\title{
INTERVENCIÓN MOTIVACIONAL EN PSICOLOGÍA DE LA SALUD: REVISIÓN DE SUS FUNDAMENTOS CONCEPTUALES, DEFINICIÓN, EVOLUCIÓN Y ESTADO ACTUAL
}

\author{
MOTIVATIONAL INTERVENTION IN HEALTH PSYCHOLOGY: A REVIEW OF THE \\ CONCEPTUAL FOUNDATIONS, DEFINITION, EVOLUTION AND CURRENT STATE
}

\author{
LUIS FlÓREZ-AlARCÓN, " HELENA VÉLEZ-BOTERO*** \\ UNIVERSIDAD NACIONAL DE COLOMBIA, BOGOTÁ - COLOMBIA \\ MARIO ENRIQUE ROJAS-RUSSELL ${ }^{* * *}$ \\ UNIVERSIDAD NACIONAL AUTÓNOMA DE MÉXICO, MÉXICO D.F. - MÉXICO
}

FECHA RECEPCIÓN: 25/06/2014 • FECHA ACEPTACIÓN: 26/09/2014

Para citar este artículo: Flórez-Alarcón, L., Vélez-Botero, H., \& Rojas-Russell, M. (2014). Intervención motivacional en psicología de la salud: revisión de sus fundamentos conceptuales, definición, evolución y estado actual. Psychologia: avances de la disciplina, 8(2), 49-71.

\begin{abstract}
Resumen
Se presenta una conceptualización acerca de la Intervención Motivacional basada en el análisis de los acontecimientos propios del Proceso Motivacional y se mencionan algunas microteorías motivacionales que proponen diversos constructos hipotéticos y variables intermediarias que explican el curso que sigue la conducta saludable en el estilo de vida de las personas, conformando lo que se considera como una Dimensión Psicológica de la Promoción y de la Prevención en Salud. Se hace un intento de definición de la Intervención Motivacional y se propone un modelo de Formulación Motivacional para darle contexto y fundamento. Se describen los temas a los que se ha aplicado la Intervención Motivacional a lo largo de la existencia formal y sistemática de este concepto, y los temas de análisis teórico y metodológico actuales, para lo cual se toman los reportes que incluyen ambos términos (Motivational, \& Intervention) en los resúmenes de publicaciones indizadas en la base de datos Medline, en el período 1991-2013.

Palabras clave: intervención motivacional, proceso motivacional, entrevista motivacional, psicología de la salud, estilo de vida.
\end{abstract}

* Psicólogo; Magíster en Análisis Experimental de la Conducta; Doctor en Psicología General Experimental. Profesor Titular, Departamento de Psicología, Grupo de Investigación «Estilo de Vida y Desarrollo Humano», Universidad Nacional de Colombia. Correspondencia: luis@florez.info

** Psicóloga, Magíster en Psicología, coordinadora del Grupo de Investigación «Estilo de Vida y Desarrollo Humano», Centro de Estudios Sociales, Universidad Nacional de Colombia.

*** Psicólogo, Maestro en Ciencias de la Salud, Doctor en Ciencias de la Salud, Profesor Titular en la carrera de Psicología, Facultad de Estudios Superiores Zaragoza, Universidad Nacional Autónoma de México. 


\begin{abstract}
A conceptualization is presented of Motivational Intervention based upon the analysis of occurrences which are part and parcel of the Motivational Process, and some motivational micro-theories are mentioned as well which propose a variety of hypothetical constructs and intervening variables accounting for the course that healthy behavior follows within the lifestyles of people, comprising what is considered to be a Psychological Dimension of Health Promotion and Prevention. An approach to the definition of Motivational Intervention is provided, and to support it and contextualize it, a model of Motivational Formulation is proposed. The topics to which Motivational Intervention has been applied throughout the formal and systematic existence of the concept are described, as well as issues of current theoretical and methodological analyses, by analyzing reports including both terms (Motivational, \& Intervention) in the abstracts of indexed publications in the Medline database, between 1991 and 2013.

Keywords: motivational intervention, motivational process, motivational interviewing, health psychology, lifestyle.
\end{abstract}

\section{Introducción}

El presente escrito se propone hacer un análisis conceptual acerca de lo que puede entenderse como Intervención Motivacional (IM), y revisar cuáles han sido sus aplicaciones al ámbito de la salud, en el marco del campo conocido como Psicología de la Salud. Como corresponde a un trabajo de reflexión teórica, eso obliga inicialmente a refrescar un conjunto de conceptos acerca del proceso motivacional, de las microteorías motivacionales sobre el comportamiento saludable, de la conceptualización misma de intervención motivacional, y, luego sí, a revisar el curso que ha seguido la evolución de esta aplicación en Psicología de la Salud.

La importancia creciente que tienen los hábitos de comportamiento propios de un estilo de vida, que los llevan a constituirse en factores sustanciales de protección de la salud o, al contrario, en factores de riesgo de enfermedad y de muerte, ha conducido en los últimos 40 años a vertebrar nuevos campos interdisciplinarios en el ámbito de la salud; estos pretenden dar cuenta del análisis científico acerca de la naturaleza biopsicosocial de los estilos de vida, y de las aplicaciones dirigidas a su manejo, control y modificación en direcciones congruentes con la promoción de la salud y con la prevención de las enfermedades. Una ilustración de estos nuevos campos es, por ejemplo, el surgimiento de la Medicina Conductual (traducción del término inglés behavioral medicine), concepto utilizado por primera vez en 1973 como subtítulo de un libro de retroalimentación biológica (biofeedback), para referirse a una modalidad de tratamientos y aplicaciones en salud asociadas al condicionamiento instrumental de respuestas fisiológicas (Birk, 1973); el término se hizo luego extensivo a todo un campo de confluencia interdisciplinaria de conocimientos sobre el comportamiento y la salud (Schwartz, \& Weiss, 1978); la existencia de una comunidad científica reunida en torno a la Sociedad Internacional de Medicina Conductual (ver http://www.isbm.info/), la realización de congresos periódicos, la existencia de publicaciones especializadas, y su inserción progresiva en el medio docente e investigativo, permiten afirmar que se trata de un paradigma particular en el abordaje científico de un campo de fenómenos de comportamiento relacionados con la salud. La propia Psicología de la Salud es otra corriente disciplinaria que se ha vertebrado en torno a la atención de todos los aspectos psicológicos relacionados con la salud y la enfermedad, dando origen a importantes aplicaciones en áreas clínicas de tratamiento y rehabilitación, así como en áreas sociales de promoción, prevención, y formulación de políticas públicas (Godoy, 1999). Otro tanto puede decirse de la Intervención Motivacional en Psicología de la Salud, término cuyo uso se propone por primera vez en este escrito. Es un término referente a un área específica de conocimientos psicológicos que, sin tener la tradición de las mencionadas antes, empieza a tener una identidad propia como área que identifica un conjunto particular de aplicaciones en el campo de la Psicología de la Salud, referente a la implementación de intervenciones fundamentadas en el análisis del proceso motivacional, con el fin de facilitar la realización de cambios en el estilo de vida de las personas, para que este sea más favorable a la salud. Con el fin de abordar la revisión de esta área, el presente escrito se divide en tres secciones, que tratan acerca del proceso motivacional y las microteorías motivacionales sobre el comportamiento saludable, de la caracterización de la intervención motivacional (IM), y de la revisión de algunas de sus aplicaciones en psicología de la salud. 


\section{El concepto de proceso motivacional y sus aplicaciones al comportamiento saludable}

Es inevitable que en la realidad actual de salud, enfermedad y muerte de nuestras poblaciones nos preguntemos por una cuestión que tiene ribetes eminentemente motivacionales: ¿Por qué la gente, a pesar de saberlo, realiza acciones que son perjudiciales e incrementan el riesgo de enfermedad, cómo podemos fomentar que las personas eviten esas acciones, y qué podemos hacer para promover que realicen otras que son benéficas para la salud y la calidad de vida? La naturaleza motivacional de la pregunta se debe a su referencia a dos aspectos definitorios de la motivación: a) De una parte, a que este proceso psicológico se refiere a lo que da inicio y mantiene los cursos de acción de la persona hacia el logro de metas. b) De otra, a que las acciones humanas que constituyen factores de protección o factores de riesgo para la salud son comportamientos complejos, voluntarios, que las personas realizan con propósitos concretos, generalmente de tipo hedonista, siguiendo secuencias conducentes a tomas de decisiones explícitas, que se pueden analizar detenidamente a partir de las fases propias del proceso motivacional. Algunos de esos análisis han llegado a tener gran relevancia en el ámbito de la salud, constituyéndose en modelos teóricos particulares que orientan la búsqueda de explicaciones y la implementación de intervenciones con el objeto de comprender y de manejar los comportamientos relevantes para la salud y para la enfermedad.

\section{El proceso motivacional}

Popularmente se considera «motivo» a aquello que mueve a la realización de algo; generalmente se trata de un estímulo que marca la presencia de una necesidad a resolverse mediante la realización de una acción deliberada de la persona. Puede ser un estímulo físico (ej. sed y la consecuente necesidad de beber), psicológico (ej. reto y la consecuente necesidad de competencia), o social (ej. evaluación y la consecuente necesidad de logro). Pero esa idea popular circunscribe el «motivo» solamente al momento de activación de un proceso, haciendo referencia apenas al impulso inicial conducente a la realización de múltiples acciones aproximatorias y consumatorias. Sin embargo, la motivación es un constructo teórico inferido, que abarca un conjunto de variables hipotéticas que se encuentran subyacentes a través del proceso conducente a la realización de dichas acciones. La idea de «proceso» implica que la motivación no es un estado único sino una sucesión de estados, cuyo análisis no se puede limitar a lo que pasa en un instante particular, ni siquiera en el instante mismo de ejecución de una acción consumatoria. Esto significa que la explicación motivacional debe abarcar integralmente lo que sucede a través del proceso motivacional, que es más amplio que la acción motivada, para que sea una explicación verdaderamente comprehensiva (Palmero, Martínez-Sánchez, \& Fernández-Abascal, 2002; Palmero et al., 2011).

La motivación es un proceso psicológico referente a lo que le da inicio a la ejecución de una acción y la mantiene en dirección al logro de un objetivo, así como a lo que determina que la acción pueda repetirse sostenidamente $o$, por el contrario, pierda fuerza en la perspectiva futura. En este sentido, se trata de un proceso eminentemente desencadenante, de dirección y de mantenimiento de la conducta (Gil-Navarro, 2007). Sin embargo, también constituye un proceso disposicional, en la medida en que las disposiciones motivacionales reflejan el impacto de la experiencia, lo que le confiere una perspectiva temporal a un determinado comportamiento como medio de acceso a un resultado esperado. La perspectiva temporal favorable, especialmente la perspectiva de tiempo pasado, consolida el comportamiento en el sentido de vida individual, por la importancia que ese comportamiento llegue a adquirir como medio de logro de una meta altamente valorada por la persona; el sentido de vida, expresado en una perspectiva de tiempo presente y de tiempo futuro, abarca las metas que son valoradas por la persona pues satisfacen sus principales necesidades en diversas áreas de su campo vital.

El carácter motivado de una conducta es un factor primordial que determina que un comportamiento pueda considerarse como una acción de la persona, en contraste con un movimiento involuntario o respuesta del organismo; en este último caso, el análisis de las respuestas autónomas del organismo es de interés prioritario para la biología, más que para la psicología. Resulta así admisible la caracterización del comportamiento, entendido como una acción con propósito, como objeto de estudio de la psicología, independientemente de su nivel de complejidad. Es 
tan acción una «simple» respuesta, como también lo es cualquier comportamiento «complejo»; por ejemplo, una respuesta como el aumento en la tasa cardiaca que sigue a la recepción de una noticia, o un comportamiento como el de un pianista que ejecuta una pieza musical; en ambos casos suceden procesos motivacionales comunes de tipo propositivo. La esencia que les confiere a ambos la calidad de «acción» es su carácter motivado en torno a un propósito. En el caso de la respuesta cardiaca ante la noticia recibida, se trata de una acción motivada que forma parte de un proceso emocional, el cual implica la evaluación cognitiva de un estímulo (la noticia recibida), y puede modificarse si cambia esa evaluación cognitiva; en el caso de la ejecución del pianista se trata de un proceso que involucra múltiples acciones cognitivas y motoras, también susceptibles de aprendizaje y cambio.

El carácter intermediario de la motivación hace referencia a su ubicación entre las situaciones o estímulos (E), externos e internos, que dan inicio al curso de una acción, de una parte, y, de otra, los comportamientos observables (R). La inferencia del proceso motivacional a partir de los comportamientos observables, y la explicación simultánea del mismo comportamiento a partir del proceso inferido constituye una especie de círculo in probando, dada la circularidad manifiesta entre los dos términos de esa relación, donde uno (la motivación), que es un constructo utilizado con pretensiones explicativas, se infiere a partir del término explicado (el comportamiento). Esta circularidad se resuelve al apelar a un conjunto de variables hipotéticas, pero que son observables en una manifestación objetiva diferente a la del mismo comportamiento bajo estudio; se trata de variables intervinientes y de constructos hipotéticos que subyacen a lo largo de las fases del proceso motivacional, tales como las expectativas, las decisiones, los planes, o las atribuciones.

El proceso motivacional es una secuencia de fases que se suceden a lo largo del curso de realización de una acción $\mathrm{R}$, desde el momento en que se percibe el $\mathrm{E}$ indicador de una necesidad que mueve el deseo a la realización de $\mathrm{R}$ (fase de activación), hasta después de realizada $\mathrm{R}$ (fase de posacción), pasando por fases intermedias en las que operan variables que mueven hacia la toma de decisión para realizar $\mathrm{R}$ (fases de predecisión y decisión), la planificación para la realización de $\mathrm{R}$ (fase de preacción), y la realización de R en sí misma (fase de acción). A estas fases les subyacen diversas variables latentes, cuyo análisis permite objetivar y operacionalizar el constructo motivacional. Son esas variables, precisamente, las que van a constituir el objetivo de la evaluación y de la intervención motivacional en psicología de la salud. La figura 1 representa este proceso, entendido como secuencia de fases con variables subyacentes; esta figura se inspira en la propuesta formulada por Heinz Heckhausen en su modelo teórico del Rubicón de las Fases de Acción (Heckhausen, \& Gollwitzer, 1987; Heckhausen, \& Heckhausen, 2008), que es una conceptualización acerca del proceso que sigue la motivación de la persona al escoger una meta, planificar y ejecutar las acciones conducentes a la meta, y, finalmente, evaluar los esfuerzos realizados y los resultados obtenidos. En la figura se representan diversas fases en el curso del desarrollo de una acción, las cuales abarcan todo el ciclo motivacional y el ciclo volitivo de la acción (Achtziger, \& Gollwitzer, 2008). Las líneas punteadas en la figura llevan a cuadros en los que se anotan los procesos subyacentes a cada fase, que son procesos cognitivos en las fases del ciclo motivacional, y una combinación de procesos cognitivos y conductuales en las fases del ciclo volitivo.

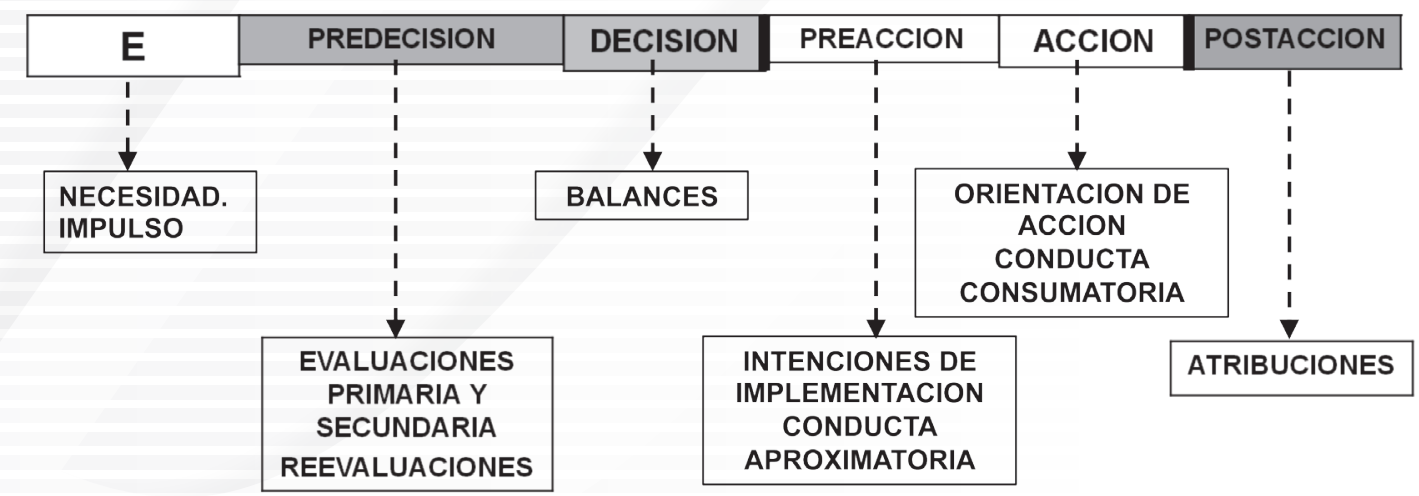

Figura 1. El proceso motivacional 
En el proceso motivacional, el ciclo motivacional propiamente dicho abarca el procesamiento de información responsable de la elección de una meta, mediante la operación de procesos cognitivos que afectan a las variables subyacentes en las fases de predecisión, decisión, y posacción (fases sombreadas en la figura 1).

Los fenómenos que suceden en un instante, así se trate del instante de realización exitosa de una acción y el fenómeno de reforzamiento que la sigue, no permiten predecir cabalmente la probabilidad de ejecución futura de la acción. Se podría suponer que la ejecución exitosa de una conducta como la de estudiar, en un momento en que esta es muy necesaria (ej. durante el periodo de exámenes), permite predecir certeramente que la persona volverá a repetirla cuando llegue el siguiente periodo de exámenes; sin embargo, la realidad podría ser muy otra si en la posacción (después de haber estudiado y de haber obtenido el resultado aprobatorio) la persona atribuye el éxito a un factor aleatorio (ej. «el examen estuvo muy fácil», «este profesor pregunta siempre lo más evidente», etc.), lo que le restaría fuerza a la expectativa de éxito fundamentada en el estudio, y así, paradójicamente, el éxito obtenido se convierte en un factor que le resta, en lugar de sumarle, a la probabilidad de repetir el comportamiento de estudio. La perspectiva futura de la motivación para estudiar, en este caso, debe incluir el análisis de lo acontecido en la pos-acción, que son las atribuciones cognitivas con las que la persona evalúa el resultado. Pero, además, también debe incluir el análisis del sentido que estudiar tiene para la persona. Si estudiar es un comportamiento que tiene gran sentido dentro de las metas vitales valoradas por la persona, su motivación para el estudio se verá menos afectada por el impacto de un éxito o de un fracaso transitorios; pero es obvio que ese sentido, a su vez, se ha construido como esquema motivacional de segundo orden sobre la base de éxitos y de fracasos anteriores.

El proceso motivacional implica también la presencia de un proceso de afrontamiento (Lazarus, \& Folkman, 1986). Las acciones motivadas R de tipo aproximatorio y de tipo consumatorio que se dan al interior del proceso motivacional, trátese de acciones motoras, fisiológicas o cognitivas, constituyen la esencia del afrontamiento que la persona realiza para resolver la necesidad desencadenada a partir de la presencia del E que da origen a la activación del proceso motivacional. El término «afrontamiento» hace referencia a la interacción entre las acciones motivadas y las necesidades que las originan; entre los comportamientos $\mathrm{R}$ concretos de la persona y la alteración de la necesidad, ya sea en un sentido de solución objetiva, como puede ocurrir cuando ese afrontamiento es instrumental, o en un sentido de modificación de la necesidad sin que haya una solución de la misma, como puede ocurrir cuando el afrontamiento es emocional. En cualquier caso, el proceso de afrontamiento (instrumental o emocional) demanda la realización de una actividad $\mathrm{R}$ por parte de la persona, en un intento de adaptarse a la situación de desequilibrio que surge con la presencia del E que señala la necesidad; el resultado adaptativo de la acción es lo que lleva a que esta se consolide (refuerce) o, por el contrario, pierda fuerza en la perspectiva temporal que regula la probabilidad de reaparición de la acción en un futuro, cuando vuelva a surgir la necesidad; dicha perspectiva depende no solo de las consecuencias objetivas de la acción, sino también de las atribuciones que la persona realiza en la fase de posacción, referentes a las relaciones de causalidad entre los esfuerzos invertidos para realizar la acción y la obtención del resultado objetivo, tema que ha sido de especial interés en el análisis propuesto por las teorías cognitivas de tipo atribucional (Weiner, 1985). Allí, en los acontecimientos atribucionales de la posacción, radica la base para la formación de una perspectiva temporal acerca de la acción, que le indica a la persona las consecuencias que puede esperar en el futuro cuando la realice (expectativa de reforzamiento-resultado). Pero esas atribuciones posacción también se van a reflejar en lo que la persona espera acerca de los esfuerzos que debe desplegar para realizar la acción (percepción de costos), en sus expectativas de autoeficacia para los mismos (controlabilidad percibida), y en la valoración de las metas que se propone alcanzar en las diversas áreas de su vida (formación de un sentido de vida).

Cualquier situación que requiera de una IM exige, por definición, que se realice un proceso de afrontamiento, en la medida en que la persona debe desplegar esfuerzos activos de autorregulación (ej. para dejar de consumir cigarrillo, para cambiar sus hábitos alimentarios, para modificar sus prácticas sexuales, para fortalecer sus hábitos de estudio, para 
incrementar la práctica de actividad física, etc.). Si la persona controla la conducta a voluntad de manera autónoma e inmediata (ej. deja de fumar cuando decide no fumar, deja de ingerir comidas inapropiadas cuando lo decide, utiliza el condón en relaciones sexuales inseguras si así lo decide, etc.), no tendría sentido poner en marcha un proceso de IM para colaborarle a modificar su comportamiento, pues ya estaría en un punto de autocontrol que no le implica asumir procesos adicionales de autorregulación para implementar un cambio de comportamiento.

El curso de cambio que sigue cualquier acción humana cuando se somete a modificación, no constituye un proceso de avance o de retroceso continuo, sino que se mueve por caminos variables que en ocasiones son congruentes y se dirigen hacia las metas previstas, pero también pueden ser incongruentes y alejarse de las mismas. En ambos casos es preciso que la persona internalice (tome conciencia acerca de) los factores que promueven el movimiento en una o en otra dirección, con el fin de adoptar las decisiones apropiadas para poder guiar su comportamiento hacia la meta prevista. En esta internalización radica la esencia autorregulatoria del proceso de afrontamiento inherente a la modificación del comportamiento que se promueve a través de la IM. «Tomar conciencia» se asume aquí como internalización progresiva de las decisiones en un proceso que conduce al fomento de la motivación autónoma y a su aumento proporcional en comparación con la motivación controlada externamente, por lo cual aquí se propone la evolución de la internalización como camino o metodología a seguir para el desarrollo de la autorregulación, adoptando algunos planteamientos que se han formulado al interior de la Teoría de la Autodeterminación (Deci, \& Ryan, 2000; Ryan, \& Deci, 2000a). En términos prácticos, esto significa que la IM debe conducir a la persona al planteamiento deliberado de procesos de afrontamiento instrumental y de afrontamiento emocional que se deben implementar en todas las fases de la modificación del comportamiento, ya sea para iniciar la ejecución del comportamiento, para mantenerlo y conducirlo a puntos más avanzados de autocontrol cuando ya se ha iniciado su ejecución, o para recuperarlo cuando se ha perdido, como sucede en los casos de recaída. La formulación motivacional que se propone más adelante como metodología para establecer los objetivos de la IM indica los focos a los cuales debe dirigirse la atención de la persona en el proceso de establecimiento de metas, mediante el señalamiento de los procesos de afrontamiento apropiados; pero es el proceso de autorregulación que se diseña para el logro de la meta el que indica la ruta de acciones que la persona debe realizar en sus esfuerzos de búsqueda activa de un objetivo específico, representado en las consecuencias inmediatas y en los resultados subsiguientes de alguna acción aproximatoria o consumatoria.

\section{Microteorías motivacionales de tipo cognitivo y comportamiento saludable}

Las aplicaciones de las teorías cognitivas sobre la motivación humana han encontrado en la promoción de la salud y en la prevención de las enfermedades un área muy fértil de trabajo, en razón de la alta importancia que los comportamientos habituales que conforman el estilo de vida de la gente tienen en la actualidad como factores de protección o como factores de riesgo para la salud. Ello ha conducido a que la IM encuentre en el fomento de la protección de la salud un campo de aplicación que ha alcanzado un alto grado de identidad en psicología, tal como lo indican los nombres de algunos modelos conceptuales contemporáneos que constituyen representaciones emblemáticas que sustentan esta modalidad de intervención, con base en los cuales se han realizado importantes contribuciones en áreas como el cumplimiento del tratamiento médico, el auto-cuidado, la actividad física, el consumo de sustancias, la alimentación, el comportamiento sexual, entre otros.

Entre los diversos modelos conceptuales en boga actualmente que sustentan de forma inmediata a la IM, algunos son de especial valor debido al alto volumen de investigaciones y de aplicaciones a las que han dado origen, como sucede con El Modelo de Creencias en Salud (Rosenstock, 1974), la Teoría de Acción Razonada (Fishbein, \& Ajzen, 1975), la Teoría de Acción Planeada (Ajzen, 1985), la Teoría de Motivación a la Protección (Rogers, 1975), el Modelo Proceso 
de Adopción de Precauciones (Weinstein, 1988), y el Modelo Transteórico (Prochaska, DiClemente, \& Norcross, 1992).

Todos los modelos motivacionales de corte cognitivo proponen constructos y variables intermediarias de gran utilidad para explicar y predecir la evolución de la probabilidad de ejecución de una acción; sin embargo, la evidencia empírica ha demostrado suficientemente que esa evolución no es una función continua que cambie de manera lineal y proporcional al estado de alguno de los factores incluidos en los modelos. Todos asumen que la toma de decisiones es un proceso que obedece a la racionalidad de la utilidad esperada por el sujeto, y que este realiza las acciones que le generan las mejores expectativas de resultado que él evalúa subjetivamente como útiles, conforme a la racionalidad propuesta por la teoría económica de utilidad subjetiva esperada (Edwards, 1954; citado por Conner, \& Norman, 2005). Sin embargo, la disparidad de los resultados encontrados en contraste con lo que se espera a partir de esta teoría (Ogden, 2003), ha llevado al planteamiento de hipótesis que consideran de gran importancia la inclusión de esos constructos y variables como factores determinantes de la elección, pero que proponen una evolución por etapas de la motivación, de tal forma que en las diversas etapas algunos de esos factores adquieren una importancia diferencial. Para ilustrar esta idea con un ejemplo, no puede suponerse que si aumenta la percepción de severidad respecto de una acción como fumar, entonces va a aumentar proporcionalmente la probabilidad de que la persona deje de hacerlo; la percepción de severidad del tabaquismo tiene un papel motivacional inicial, pero luego entran a jugar un papel determinante otros factores que adquieren por momentos mayor significación, como la percepción de vulnerabilidad, la percepción de autoeficacia, las normas subjetivas acerca del tabaquismo, etc. Surgen así los modelos de etapas (Sutton, 2005), que representan una fase nueva de los conceptos motivacionales, que tiene un especial impacto en las aplicaciones que se realizan en el campo de la salud; Sutton destaca entre estos modelos la relevancia que han tenido el MTT, el MPAP, y la Aproximación de Procesos a la Acción Saludable (HAPA por su sigla en inglés).

En un intento de dar coherencia al complejo campo de propuestas cognitivas acerca de los factores subyacentes al desarrollo de la motivación para la ejecución de una conducta protectora, Flórez (2007) propone un heurístico al que denomina Dimensión Psicológica de la Promoción y de la Prevención en Salud. No se trata de un modelo nuevo, sino de un esquema que representa una nueva visión de las etapas (ver la figura 2), no ya como momentos fijos y necesariamente secuenciales, sino como fases en las que se ubican barreras (B) cuya superación tiene una importancia sustancial para la superación de cada fase; entre los factores que constituyen dichas barreras se destacan las expectativas de reforzamiento-resultado (B1), las expectativas de controlabilidad (B2), las actitudes normativas (B3), la toma de decisiones (B4), y la planificación de las acciones (B5). En la medida en que transcurren estas fases, de acuerdo con esta visión del proceso motivacional, van surgiendo nuevos objetivos para el proceso autorregulatorio conducente a la acción, cuyo cumplimiento aumenta la disponibilidad al cambio y, con ello, aumenta la probabilidad de la acción, la cual puede suceder en cualquier instante, no solo en la fase final, aunque esa probabilidad es menor en las fases anteriores. En otras palabras, este heurístico considera que en cualquier fase del proceso de desarrollo de la motivación puede suceder la acción, pero le asigna una probabilidad menor de desembocar en la acción (incumplimiento sistemático) a lo que sucede en las primeras fases, y una mayor probabilidad de concluir en la acción (cumplimiento sistemático) a lo que sucede en las fases ulteriores, especialmente cuando se concluye la fase de toma de decisiones con balances favorables a la percepción de beneficios de la acción, en relación con la percepción de costos de la misma. La dimensión psicológica de la promoción y de la prevención en salud ha inspirado diversas experiencias de promoción de la salud en el ámbito escolar (ver www.tipica.org). 


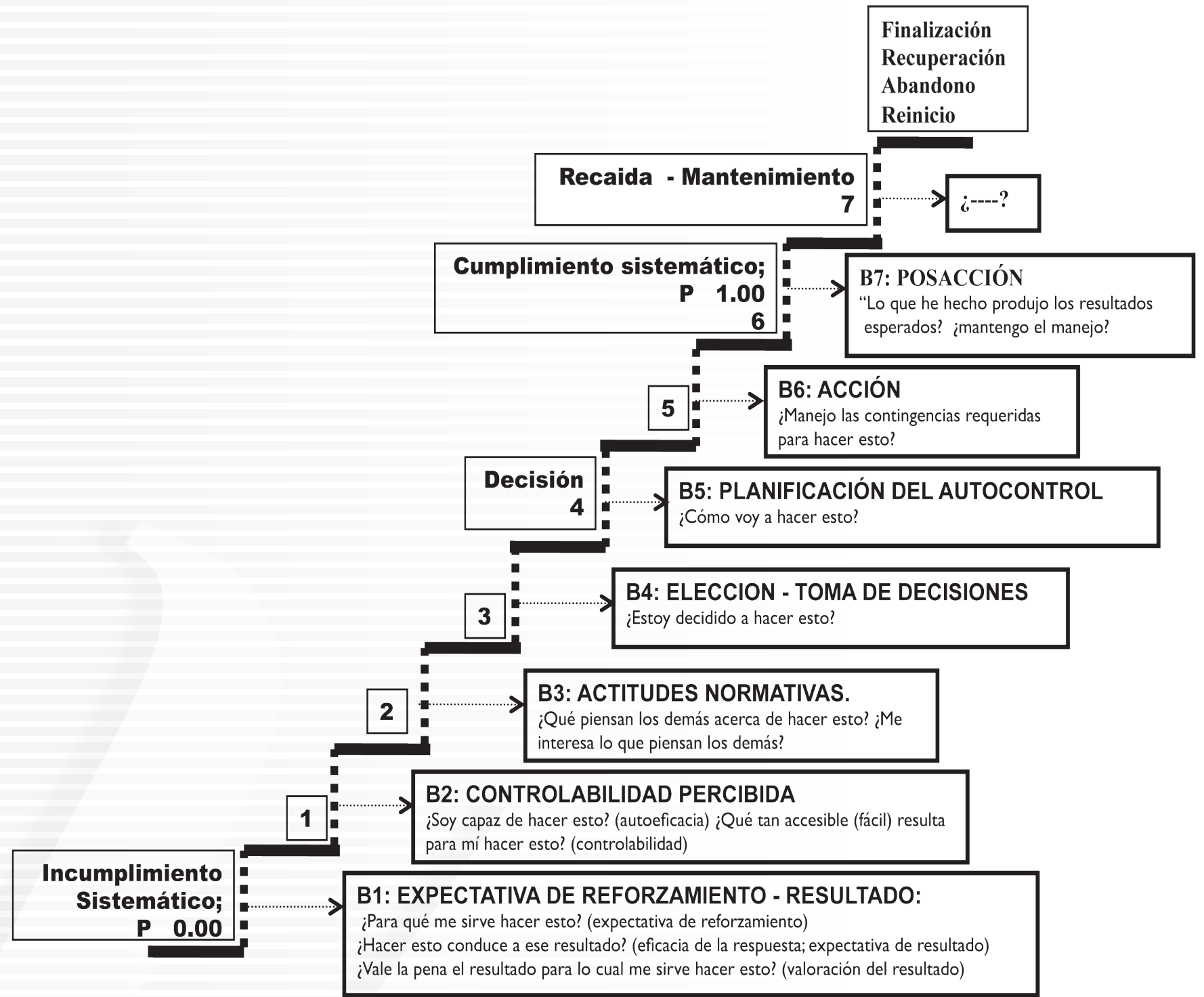

Figura 2. Dimensión psicológica de la promoción y de la prevención en salud

\section{Conceptualización de la intervención motivacional}

El uso del término «Intervención Motivacional» (IM) empezó a hacerse frecuente en la década de los años 90 durante el siglo XX para caracterizar una modalidad de tratamientos aplicados a personas que presentan excesos conductuales, especialmente excesos en el consumo de sustancias psicoactivas, sin configurar aún situaciones de dependencia caracterizadas por la presencia del síndrome de abstinencia. Se trataba de una nueva conceptualización que, a diferencia de los conceptos tradicionales sobre terapias de autocontrol, que enfatizan sobre el manejo de contingencias hecho por la propia persona, venía a enfatizar sobre la modificación de los factores subyacentes al proceso motivacional como condición que lleva a la persona a presentar variaciones en la disponibilidad que manifiesta hacia la ejecución de un comportamiento particular, como es el consumo de una sustancia. Aunque las denominaciones oscilaron entre términos como «entrevista motivacional» (Miller, \& Rollnick, 1991), 
«terapia de fortalecimiento motivacional» (Miller, Zweben, DiClemente, \& Rychtarik, 1995) y «consejería motivacional» (Klinger, \& Cox, 2004), a finales de esa década ya se usaba con más propiedad el término Intervención Motivacional Breve para denominar a estos tratamientos utilizados en psicología de la salud (Craig, Hungerford, \& Dunn, 2005), uso que se consolida en la primera década del siglo XXI, extendiéndose su aplicación al manejo de múltiples comportamientos relacionados con la salud, y no solo a los de consumo de sustancias.

Denominacionescomo «Intervención Motivacional» e «Intervención Motivacional Breve» corresponden más a una metodología que a un procedimiento específico; eso ha llevado a que su utilización se haya realizado con referencia a procedimientos diversos, lo cual genera algún grado de ambigüedad y de dificultad al comparar reportes que usan el mismo término sin que lo hecho en unos estudios corresponda necesariamente con lo realizado en otros. Sin duda, el procedimiento más representativo de la IM es la Entrevista Motivacional (Miller, \& Rollnick, 1991), pero no siempre lo que se reporta como IM se atiene a los criterios propios de este procedimiento. Esta realidad condujo a Rollnick et al. (2002), a referirse a tres modalidades distintas de intervención, clasificación que ellos hacen con el objeto de preservar la identidad y la integridad estructural de la entrevista motivacional, distinguiéndola de otras adaptaciones que no cumplen con todas las condiciones metodológicas propias de ese procedimiento: a) consejo breve, donde el interventor busca informar sobre los riesgos y dar inicio a secuencias de cambio conductual; b) consejería para el cambio conductual, donde el interventor cumple una función de escucha empática evitando la confrontación de la resistencia, tratando de comprender al consultante, pero sin dirigirlo expresamente a la solución de la ambivalencia frente al cambio; y, c) entrevista motivacional, donde el interventor sigue expresamente los principios de generación de empatía, fomento de autoeficacia, nodiscusión, uso de la aceptación con un giro y de la reformulación, y fomento de la discrepancia, todos con el fin de incrementar la motivación y la disponibilidad al cambio, y la solución de la ambivalencia. Entre estas tres modalidades los autores establecen diferencias en función del contexto y las metas de la intervención, así como del estilo de la interacción entre interventor y consultante, y de las habilidades de intervención requeridas en cada caso.

Para caracterizar lo que sí es y lo que no es IM, a juicio de los autores del presente escrito, más que establecer la presencia de diferentes modalidades de práctica en el marco de la IM, lo cual es incuestionable, debe procurarse establecer cuáles son las condiciones que le confieren identidad al campo de la IM. El concepto de intervención motivacional hace referencia a los intentos de modificación del comportamiento a partir de la afectación de diversas variables intermediarias y constructos hipotéticos subyacentes en las diversas fases del proceso motivacional. Sin embargo, no a cualquier intento de modificación de dichos factores se le puede asignar el status de IM, sino solamente a los que cumplan con algunos criterios que se incluyen en su definición, y que, además, sigan una metodología sistemática que, de forma genérica, aquí se analiza como un proceso de formulación motivacional.

\section{Un intento de definición}

La IM es una metodología sistemática dirigida a la modificación de los factores subyacentes al proceso motivacional, con el fin de fomentar el afrontamiento emocional y el afrontamiento instrumental requerido para la realización de acciones que resuelvan alguna necesidad relevante para la persona; se puede poner en práctica mediante la aplicación de protocolos de intervención breve, que incorporan diversos procedimientos de evaluación y de tratamiento cognitivos y conductuales que son coherentes con ella, en situaciones de normalidad que descartan la presencia de patologías biológicas o psicológicas interferentes con el curso autorregulado de las acciones por parte de la persona, cuyos resultados son susceptibles de evaluación objetiva. Esta conceptualización contiene diversos términos que apuntan a aspectos fundamentales de la IM, caracterizando su postura epistemológica, teórica y metodológica como parte de la ciencia psicológica, entre los cuales se destacan algunos términos relevantes que se incluyen en el cuadro 1, con intenciones de clarificar el significado atribuido a cada uno de ellos. 


\section{La formulación motivacional}

La función esencial de una formulación es proponer alguna hipótesis acerca de cuáles son los factores y los mecanismos psicológicos que originan y mantienen un problema, con el fin de orientar la evaluación y el tratamiento del caso a partir de dicha hipótesis. Persons y Davidson (2010) proponen que la formulación que guíe el abordaje de un caso en el marco de una intervención de tipo cognitivo-conductual, como lo es la IM, debe cumplir funciones de orientación que se reflejen en los procesos de evaluación y diagnóstico, planificación del tratamiento, implementación del tratamiento, y evaluación de los resultados; en el caso de la IM estas funciones que debe cumplir la formulación pueden entenderse de la siguiente manera:

1) Orientar la evaluación inicial, para concluir en un diagnóstico: Este diagnóstico es en esencia una descripción del estado en que se encuentra el proceso motivacional circundante a un comportamiento particular ubicado en el epicentro de una situación problema para el individuo, tal como el proceso motivacional se asume al interior de algún modelo teórico particular que se utilice. En una ilustración, supóngase que la situación problema de una persona sea una problemática de salud ocasionada por sobrepeso corporal, en la cual intervienen como comportamientos centrales la ingesta de alimento y la actividad física; si se limita la ilustración al comportamiento de actividad física, bajo el supuesto que la IM del caso se orienta conforme a la propuesta de la Teoría de Motivación a la Protección, se deberían observar (medir) y evaluar fenómenos y relaciones como las siguientes: a) El comportamiento sedentario (especialmente sus beneficios); b) el comportamiento de actividad física (topografía, frecuencia, duración, intensidad, etc.); c) las percepciones de severidad del daño asociado al sedentarismo; d) las percepciones de susceptibilidad personal al daño generado por el sedentarismo; e) las percepciones de eficacia y de autoeficacia asociadas a la práctica de la actividad física; f) las percepciones de costo asociadas a la práctica de la actividad física; g) la evaluación de amenaza: relación entre beneficios y daños asociados al sedentarismo; h) la evaluación de afrontamiento: relación entre eficacia y costos asociados a la actividad física; i) la motivación hacia la práctica de la actividad física (disponibilidad al cambio); j) las consecuencias inmediatas y los resultados mediatos de la actividad física (ej. medidas antropométricas, peso corporal, aspectos subjetivos de la calidad de vida, etc.).

El diagnóstico debe entenderse como una hipótesis sobre los mecanismos motivacionales subyacentes al comportamiento, y no como una categoría perteneciente a un sistema clasificatorio de desórdenes mentales o del comportamiento. En la anterior ilustración podrían ser mecanismos hipotéticos que subyacen a la baja práctica de la actividad física: a) Una baja comprensión de sus beneficios potenciales; b) una deficiente explicación de resultados negativos anteriores, a partir de atribuciones incorrectas; c) una alta relación costo-beneficio a partir de magnificaciones del valor del sedentarismo; d) una baja confianza en las propias capacidades para comprometerse exitosamente en la práctica de un plan de actividad física; e) una deficiente planificación de alternativas realistas para superar obstáculos objetivos a la hora de realizar la actividad física. La formulación debe proponer cuáles mecanismos se van a afectar con la IM, el orden en que se van a abordar, y, sobre todo, cuál es el proceso de afrontamiento que se busca impulsar.

2) Orientar la elaboración del plan de tratamiento: el plan del tratamiento es la especificación de los factores y de los mecanismos psicológicos que se proponen afectar mediante la IM, con el fin de obtener los resultados de cambio en el comportamiento del consultante, en coherencia con la hipótesis diagnóstica que se haya concluido en la fase de evaluación. En el caso de la ilustración, el plan podría ser, por ejemplo, fortalecer la evaluación de afrontamiento, la toma de decisiones, y la planificación de las acciones. Para eso podrían plantearse como objetivos: a) Fortalecer la percepción de autoeficacia; b) restarle valor a las percepciones de costo asociadas a la actividad física; c) favorecer la toma de decisiones específicas para la práctica de la actividad física; d) fomentar la elaboración de algunos planes específicos que, a la manera de intenciones de implementación, le permitan a la persona llevar a la práctica la decisión tomada; y, e) modificar un esquema nuclear de incompetencia personal 
del sujeto, inferido por el interventor como mecanismo central de diátesis que predispone a los pensamientos automáticos expresados por la persona en relación con su baja autoeficacia para la práctica de la actividad física y con los beneficios del sedentarismo.

3) Orientar la selección e implementación de estrategias de tratamiento: es la selección de algunos procedimientos particulares de IM que permitan movilizar los procesos de cambio requeridos a fin de afectar los factores y los mecanismos señalados en la formulación. El campo de la terapia cognitivoconductual (Dobson, 2010) aporta un conjunto de técnicas o procedimientos de intervención susceptibles de adaptación para ser utilizados a fin de lograr los objetivos que se propongan en un caso de IM. Algunos de esos procedimientos son más afines con los propósitos de la IM. En el caso hipotético de la ilustración podría adoptarse un procedimiento de diálogo socrático para alterar los pensamientos automáticos y las distorsiones cognitivas presentes en las autoafirmaciones de la persona acerca de su autoeficacia y de los costos inherentes al abandono del sedentarismo, así como para modificar un esquema nuclear de baja competencia relacionado con los anteriores pensamientos. También podría implementarse un procedimiento de entrevista motivacional para fomentar el avance del proceso de toma de decisiones. Y un procedimiento de autocontrol como SCIENCE para poner en práctica un plan de acciones conducentes a la meta decidida (Mahoney, \& Mahoney, 1981). Aunque es muy importante tener claridad acerca de los procedimientos o técnicas que se van a utilizar, a fin de hacerlo conforme a los requerimientos que exige una adecuada implementación, antes es muy importante tener claridad acerca de los procesos motivacionales de cambio a los cuales sirven dichas técnicas o procedimientos.

4) Orientar el monitoreo continuo y el sometimiento a prueba de las hipótesis: es la observación de algunos aspectos, como: a) Observación de los cambios en los factores y mecanismos psicológicos postulados como causa del comportamiento; b) observación de los cambios consecuentes que ocurren concomitantemente en dicho comportamiento, el cual forma parte de un proceso de afrontamiento emocional o de afrontamiento instrumental; c) observación de las consecuencias de esos cambios comportamentales y de su impacto sobre aspectos relevantes para juzgar la adaptación vital de la persona a corto, mediano y largo plazos. En el caso hipotético de la ilustración que se ha venido manejando se espera que un aumento de las expectativas de autoeficacia para la práctica de la actividad física y una reducción de las expectativas de beneficio frente al sedentarismo (aspecto «a» señalado antes) conduzcan a una toma explícita de decisiones sobre realización de actividad física (aspecto «b»). Igualmente se espera que la adopción de algunas intenciones de implementación favorezca a corto plazo el mantenimiento de la persona en la práctica de la actividad. A mediano plazo se espera el cambio en indicadores biológicos de sobrepeso, como la reducción del índice de masa corporal (aspecto «C»). A largo plazo pueden esperarse resultados como un incremento en la resistencia física de la persona, y una reducción de síntomas de enfermedad asociados al sobrepeso, tales como los indicadores de glicemia y otros que señalen el avance hacia la solución del problema de salud que llevó a la persona a involucrarse en un plan de modificación del sobrepeso corporal. El monitoreo de los indicadores relacionados con los anteriores resultados va a ser un elemento de información de retorno fundamental para que la persona mantenga las decisiones y los planes de implementación adoptados, o los modifique como resultado de la evaluación realizada, hasta llegar a un punto de finalización del plan, que corresponda al surgimiento de un nuevo estado autocontrolado de prácticas habituales (en este caso de prácticas de actividad física) y de consecuencias adaptativas de estas prácticas que mejoren la calidad de vida de la persona, al facilitarle la realización de metas que forman parte importante de su sentido de vida.

Un esquema de formulación que puede orientar las intervenciones requeridas para el cumplimiento de las funciones reseñadas antes, es el que se ilustra en la figura 3. Se trata de un esquema inspirado en el modelo de formulación cognitiva por niveles que propone Riso (2006). En este esquema de formulación motivacional se destacan los siguientes elementos: 


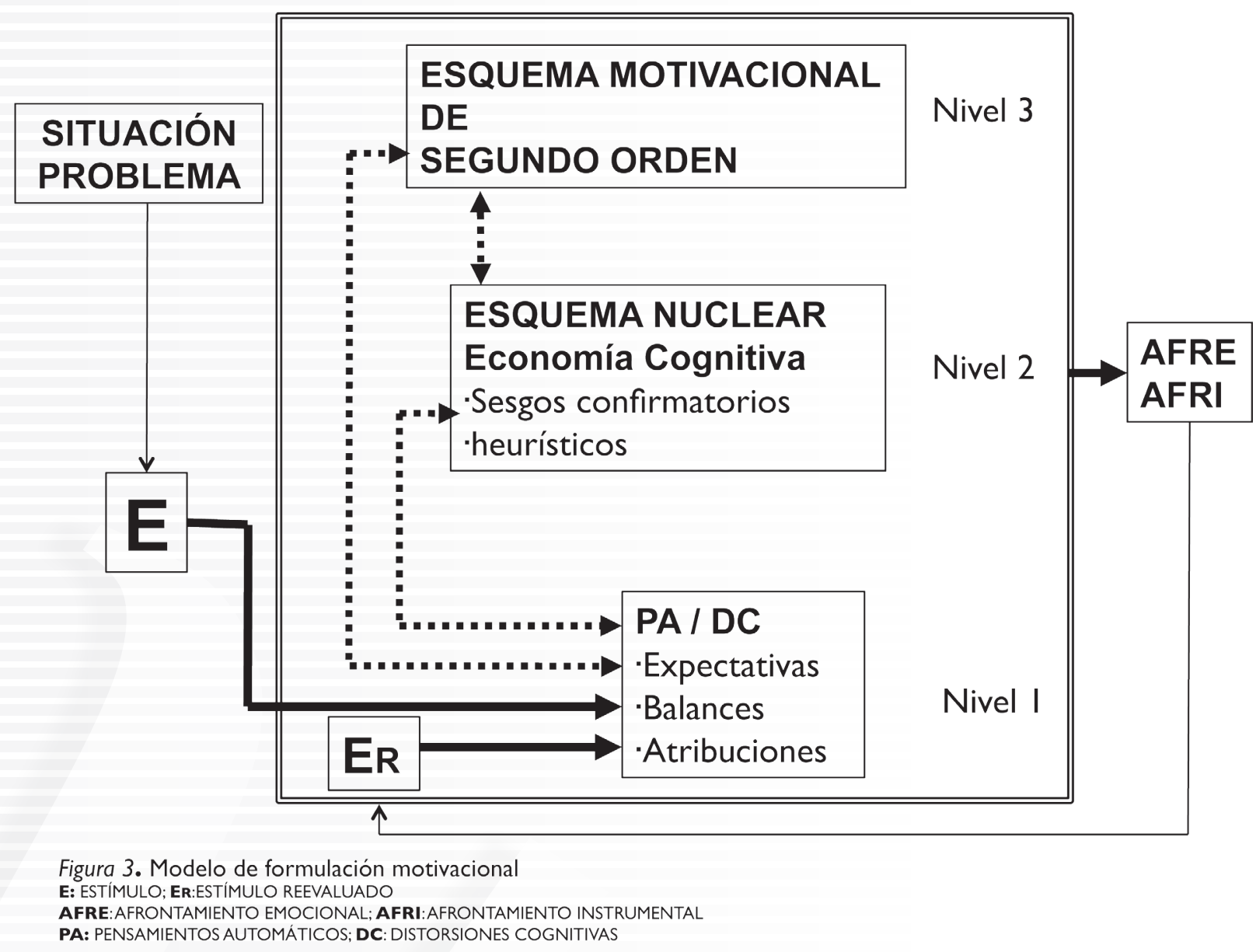

Situación problema: Es el contexto medioambiental explícito en el que se enmarca la problemática de salud que da origen a la intervención motivacional en psicología de la salud. Puede ser una situación de sobrepeso corporal que representa un riesgo de enfermedad, el consumo de una sustancia, el cumplimiento de un tratamiento médico, el monitoreo de un indicador de enfermedad, la adopción de un comportamiento de autocuidado, etc. En ese contexto es preciso establecer los estímulos externos e internos (E) que constituyen aspectos relevantes en el análisis del comportamiento específico en cuestión, los cuales adquieren relevancia en función de su papel como estímulos discriminativos del comportamiento, que desencadenan el proceso motivacional relacionado con el exceso o con el déficit conductual de que se trate (exceso de consumo de alimentos, déficit de actividad física, déficit de respuestas emocionales adaptativas, déficit de habilidades sociales, exceso de consumo de sustancias, etc.).

Procesamiento de información: Es el bloque central de procesamiento cognitivo y afectivo de la información que, a manera de variable intermediaria, se ubica entre la situación problema antecedente y el proceso de afrontamiento consecuente. En ese bloque se distinguen tres niveles diferentes del procesamiento de la información, cuyas características se sintetizan en el cuadro 2: El nivel 1, que es el nivel del «motivo» propiamente dicho, el nivel 2, que corresponde a la perspectiva que la persona se ha formado en el pasado acerca de la situación problema, y el nivel 3, que 
corresponde al encuadre de la situación problema dentro del sentido de vida de la persona.

Proceso de afrontamiento: La interacción entre los tres niveles del procesamiento de información arroja como resultado un proceso de afrontamiento, ya sea este de predominio instrumental o emocional, el cual incorpora un curso de acciones motivadas, aproximatorias o consumatorias, directamente relacionadas con los déficits o con los excesos comportamentales. Estas acciones pueden modificar objetivamente la situación problema (afrontamiento instrumental) o su significación personal (afrontamiento emocional), con lo cual le aportan nuevos niveles de adaptación a la persona, cuya significación subjetiva en términos de atribuciones personales, u objetiva en términos de calidad de vida, va a conducir a que evolucione la reevaluación que la persona hace acerca de las relaciones entre el comportamiento y sus consecuencias, con las consecuentes transformaciones en la percepción de los E y en su evaluación cognitiva, en un sentido que, se espera, conduzca a grados mayores de autorregulación en la dirección adaptativa (nuevas prácticas alimentarias, nuevas prácticas de actividad física, moderación en el consumo, mayor auto-cuidado, mejores habilidades, etc.).

El esquema de formulación no es estático sino dinámico. Refleja el estado de un bloque central de procesamiento informacional en un momento inicial, y su evolución a medida que se va realizando la intervención. Al final de la misma, debe reflejar un estado del proceso de afrontamiento correspondiente a etapas motivacionales más avanzadas, en consonancia con los avances logrados en el proceso motivacional, y con los avances en el proceso de autorregulación del comportamiento, resultante de una mayor internalización de nuevos esquemas, más adaptativos, en los tres niveles de la formulación. La IM puede corresponder a una acción instantánea, p. ej. La emisión de un mensaje a través de medios masivos, cuyas características estimulares se diseñan en correspondencia con la estructura cognitiva de los receptores potenciales, población «blanco» $\mathrm{o}$ «target» al cual va dirigido el mensaje (Galvis, 2013). O puede corresponder a una intervención grupal, diseñada en función de una característica común del grupo de receptores, tal como la identidad en la etapa motivacional respecto al comportamiento que se espera afectar al final. Lo más importante es que este esquema de formulación motivacional debe orientar las acciones de evaluación y de tratamiento que se realicen en el marco de la intervención motivacional en psicología de la salud.

\section{Aplicaciones de la intervención motivacional en psicología de la salud}

En el ámbito de la salud, la IM ha encontrado un nicho natural de aplicaciones en el campo de la educación para la salud, específicamente en áreas de prevención universal, de prevención selectiva, y de prevención indicada, cuando se realizan prácticas de promoción de la salud y/o de prevención de las enfermedades; a esas aplicaciones de la IM se hace referencia en el presente texto como «intervención motivacional en psicología de la salud». Ya que el objetivo del presente escrito no es hacer un meta-análisis acerca de las aplicaciones de la intervención motivacional en psicología de la salud, sino revisar cuáles han sido las aplicaciones de la IM en el ámbito de la salud, se hace una revisión de publicaciones reportadas en una base de datos típica de este ámbito, la base de datos Medline. Para hacerlo se utilizó el descriptor «intervención motivacional» (motivational intervention) en el resumen de los artículos. La revisión se realizó en dos partes: a) una referente al origen y la evolución en las dos primeras décadas de uso del término IM, entre los años 1991 y 2010 ; y b) su estado actual, para lo cual se revisan los artículos publicados en la misma base de datos entre 2011 y 2013.

\section{Origen y evolución inicial de la intervención motivacional en psicología de la salud (1991-2010)}

La revisión de antecedentes realizada en Medline para el período 1991-2010, arrojó un total de 445 estudios; esta revisión fue hecha por Flórez-Alarcón (2013) con el propósito de sustentar el carácter innovador de la IM; al depurar la revisión, se retiraron 31 estudios que no corresponden al área de la salud, incluidos algunos de meta-análisis, con lo cual se hizo el análisis sobre un total de 414 reportes, cuyas frecuencias por tema y por período se presentan en la Tabla 1. 
Tabla 1. Revisión de estudios que incluyen en el abstract los descriptores «Motivational»e «Intervention» en la base MEDLINE entre los años 1991 y 2010 (entre paréntesis: porcentaje del total de estudios del periodo)

\section{ESTUDIOS PUBLICADOS POR PERIODO}

\begin{tabular}{|c|c|c|c|c|}
\hline & 1991-1995 & $1996-2000$ & 2001-2005 & 2006-2010 \\
\hline Alcohol & $3(50 \%)$ & $12(38,7 \%)$ & $26(27,7 \%)$ & $47(16,6 \%)$ \\
\hline Sexo riesgoso & $1(16,6 \%)$ & $2(6,45 \%)$ & $4(4,25 \%)$ & $21(7,42 \%)$ \\
\hline Entrenamiento a padres & $1(16,6 \%)$ & $2(6,45 \%)$ & $3(3,19 \%)$ & $2(0,71 \%)$ \\
\hline Adhesión al tratamiento & $1(16,6 \%)$ & $7(22,6 \%)$ & $13(13,8 \%)$ & $41(14,5 \%)$ \\
\hline Comportamiento alimentario & & $1(3,22 \%)$ & $3(1,06 \%)$ & $9(1,06 \%)$ \\
\hline Tabaquismo & & $1(3,22 \%)$ & $11(11,7 \%)$ & $40(14,1 \%)$ \\
\hline Drogas & & $4(12,9 \%)$ & $19(20,2 \%)$ & $36(12,7 \%)$ \\
\hline Obesidad o sobrepeso & & $1(3,22 \%)$ & $1(1,06 \%)$ & $10(3,53 \%)$ \\
\hline Autoprotección & & $1(3,22 \%)$ & $4(4,25 \%)$ & $11(3,89 \%)$ \\
\hline Actividad física & & & $8(8,5 \%)$ & $36(12,7 \%)$ \\
\hline Juego patológico & & & $1(1,06 \%)$ & $3(1,06 \%)$ \\
\hline Estilo de vida & & & $1(1,06 \%)$ & $21(7,42 \%)$ \\
\hline Violencia & & & & $6(2,12 \%)$ \\
\hline Total de estudios por periodo & 6 & 31 & 94 & 283 \\
\hline Porcentaje del gran total & $1,4 \%$ & $7,5 \%$ & $22,7 \%$ & $68,4 \%$ \\
\hline
\end{tabular}

La tabla 1 permite apreciar con bastante claridad el incremento progresivo de los estudios sobre IM entre los años 1991 y 2010. Al describirlos por períodos, en el primer quinquenio solamente se publicaron 6 estudios con ese descriptor, cifra que aumentó a 283 publicaciones en el último quinquenio. Igualmente es notable la ampliación de las temáticas a las cuales se refieren estos estudios, a medida que se avanza en quinquenios: en los primeros años en que se empezó a realizar la aplicación, el tema central fue el de alcohol, particularmente en prácticas de prevención selectiva e indicada del abuso alcohólico con poblaciones escolares y universitarias, no así de tratamiento de la dependencia alcohólica. Luego los dos temas que fueron incrementando su presencia progresiva fueron el de modificación del sexo riesgoso, especialmente en poblaciones de alto riesgo de adquisición de VIH, y el de adhesión al tratamiento médico en la atención de diversas enfermedades, especialmente de diabetes y de enfermedades cardiovasculares. Los estudios sobre entrenamiento a padres incluyen especialmente educación en prácticas de cuidado a padres de niños con problemas de desarrollo. A partir del segundo quinquenio empiezan a hacerse extensivos los métodos aplicados al control del consumo excesivo de alcohol para aplicarlos al consumo de cigarrillo y de otras sustancias ilícitas («drogas»), especialmente marihuana. Los estudios sobre comportamiento alimentario hacen referencia a cambio en prácticas alimentarias en sujetos que no 
tienen sobrepeso, con la intención de mejorar la calidad de sus dietas, mientras que los estudios sobre control de peso hacen referencia a personas con sobrepeso o con obesidad, e incluyen simultáneamente modificación de hábitos alimentarios y de actividad física. Este último tema muestra un incremento significativo de frecuencia en el último quinquenio, como medio de prevención universal, sin relacionarse específicamente con alguna enfermedad. La autoprotección se refiere especialmente al monitoreo de síntomas para detección precoz de alguna enfermedad, pero también a otras conductas como vacunación y uso del cinturón de seguridad para evitar lesiones en casos de accidentalidad. El estilo de vida se refiere a modificación simultánea de varios hábitos de comportamiento que constituyen factores de riesgo o de protección. El juego patológico («adicción al juego») y la modificación de algunos comportamientos de violencia familiar aparecen especialmente en el último quinquenio.

La relación entre estudios referentes a temas de consumo de sustancias psicoactivas y estudios sobre otros temas de salud en las aplicaciones de la IM, es favorable al primer tema en los tres primeros quinquenios de las dos últimas décadas; pero se invierte en el último quinquenio (43\% temas de sustancias versus $57 \%$ temas de salud), tendencia que va a reafirmarse en los años siguientes.

En cuanto a la modalidad de intervención practicada, los reportes favorecen ampliamente a la Entrevista Motivacional, pero también se refieren a la Terapia de Fortalecimiento Motivacional, la Consejería Motivacional, y la Intervención Motivacional Breve, los cuales se han practicado en contextos de intervención individual y de intervención grupal. La denominación «Intervención Motivacional Breve» tiende a aparecer con mayor frecuencia en los últimos años, y puede incluir (o no) la práctica de la entrevista motivacional, de procedimientos de autorregulación del comportamiento, y de procedimientos de reestructuración cognitiva; por lo anterior se sugiere la adopción de un sistema formal de formulación motivacional, como el que se propuso antes aquí, que constituya un sistema metodológico más amplio que la práctica de algún procedimiento particular, como criterio esencial para caracterizar las aplicaciones que se vayan a incluir bajo el rótulo de «Intervención Motivacional».
Algunas conclusiones que pueden extraerse de la revisión de estudios hecha en la base Medline para este período son:

1. La denominación «Intervención Motivacional» empieza a hacerse frecuente en los estudios psicológicos a partir de los primeros años de la década 1990, especialmente para referirse a aplicaciones realizadas con el propósito de prevenir o de revertir el consumo excesivo de alcohol en poblaciones no-clínicas, particularmente en el medio escolar.

2. A partir de la primera gran aplicación al consumo de alcohol, la IM se fue haciendo paulatinamente extensiva a la prevención y a la modificación del consumo de otras sustancias psicoactivas, especialmente de cigarrillo y de marihuana.

3. En el ámbito de la salud la práctica de la IM se ha aplicado en asociación a la importancia que tiene la modificación de hábitos de comportamiento en la prevención y el tratamiento de enfermedades crónicas, y para el fomento de la adhesión a los tratamientos médicos, especialmente cuando incluyen modificaciones en el estilo de vida.

4. Las aplicaciones de la IM abarcan poblaciones, procedimientos, y metodologías diversas y crecientes en complejidad, pero esa misma diversidad plantea la necesidad de unificar criterios y denominaciones que permitan caracterizar esta práctica con mayor grado de precisión, a fin, también, de poder reportar con mayor claridad lo que indican las evaluaciones acerca de su impacto y de sus alcances.

5. La IM tiene un respaldo teórico muy amplio para hacer su extensión a la salud; este respaldo se expresa especialmente en las denominadas microteorías cognitivo-sociales y de etapas acerca del comportamiento saludable.

\section{Estado actual de la intervención motivacional en psicología de la salud (2011-2013)}

Para analizar el estado actual en el desarrollo de la intervención motivacional en psicología de la salud se revisaron en la misma base Medline las publicaciones que incluyen el descriptor Motivational Intervention en el período comprendido entre 2011 y 2013, obteniéndose los temas y las frecuencias que se sintetizan en la tabla 2. En esta tabla se ordenan los temas tratados, de mayor a menor frecuencia. 
Tabla 2: Frecuencia de publicaciones que incluyen en el abstract los descriptores «Motivational» e «Intervention», reportadas en la base de datos Medline, en el período comprendido entre 2011-2013

\section{TEMA}

Programas grupales de promoción y de prevención

Adherencia al tratamiento

Teórico-metodológicos

Control de peso

Actividad física

Alcohol

Drogas

Tabaquismo

Sexo riesgoso

Autoprotección

Dieta

TOTAL

\section{FRECUENCIA}

65

34

33

32

26

25

21

20

15

6

2
PORCENTAJE DEL TOTAL

23,3

12,2

11,9

11,5

9,3

8,9

7,5

7,2

5,4

2,1

0,7
Aunque el período abarcado por la tabla 2 es de solo tres años, se puede observar que la cantidad de publicaciones reportadas (279) es prácticamente igual al del período anterior en la tabla 1 (283), que era de cinco años, lo cual refleja el incremento sostenido en el número de investigaciones que tratan sobre temas de intervención motivacional en psicología de la salud. Se resaltan tres diferencias del período actual, con el período anterior en la evolución de estas aplicaciones:

1) El incremento proporcional de inclusión de temáticas diferentes a las de consumo de sustancias (alcohol, tabaco y drogas). Estas temáticas de consumo, que antes eran notablemente privilegiadas por la IM, ahora ocupan solo una cuarta parte del total de las investigaciones reportadas, pasando a tener una mayor presencia otras temáticas de salud, todas muy relacionadas con comportamientos relevantes en el campo de las enfermedades crónicas no transmisibles.

2) Hay una tendencia notable a la investigación sobre los resultados y el impacto de la IM mediante la realización de aplicaciones dirigidas a grupos poblacionales específicos, reunidos en función de características sociales y demográficas (ej. escolaridad, etnia, género, etc.) con intenciones de hacer promoción de la salud y prevención universal (en especial de la salud mental), así como prevención selectiva e indicada de desórdenes específicos (de salud oral, de desórdenes cardiovasculares, de diabetes, de enfermedades respiratorias, etc.). La mayor parte de estas investigaciones siguen diseños cuasi-experimentales, con grupos de control sin intervención, y se dirigen especialmente a evaluar la eficacia de la IM.

3) Empiezan a aparecer estudios con intenciones de análisis teórico y metodológico que, además de sintetizar los logros característicos de la aplicación en un campo particular, establecen comparaciones entre diversas modalidades de IM en un mismo campo, evalúan cuáles son los factores motivacionales específicamente responsables de los cambios, establecen comparaciones entre análisis retrospectivos y prospectivos, inspeccionan los aspectos de formación o habilidad que deben cumplir los terapeutas, analizan la coherencia entre los modelos conceptuales subyacentes a las intervenciones y la estructura de las mismas, y tratan sobre asuntos éticos en las aplicaciones de la intervención motivacional en psicología de la salud. 


\section{Discusión}

La IM tiene una corta historia, de aproximadamente 25 años, como área de estudio sistemático en el campo más amplio de la psicología de la salud; sus orígenes se identifican con la propuesta de entrevista motivacional que hicieron Miller y Rollnick (1991), pero su aplicación se ha expandido con el paso de los años hacia el uso de un acervo más amplio de técnicas, lo cual justifica que se hagan intentos más precisos de conceptualización y definición de lo que se entiende por IM. Su característica central ha sido el intento de abordar la explicación, análisis y transformación del comportamiento saludable a partir de categorías referentes al proceso motivacional, tratándolo desde una perspectiva de normalidad y no desde una perspectiva psicopatológica. Este hecho ha favorecido que la IM se apoye en la investigación básica sobre la motivación humana en general, la cual ha conducido al planteamiento de algunos modelos conceptuales que intentan sistematizar el conjunto de factores esenciales que son responsables del estado actual de la probabilidad de ejecución de un comportamiento específico, que adquiere relevancia como factor de riesgo o de protección en un contexto de salud particular. Dichos modelos se han aplicado con diverso grado de creatividad y de efectividad a la solución de problemas de gran importancia en el campo de la salud, como son los problemas de adhesión a los tratamientos, de uso responsable de los servicios de salud, de modificación de los comportamientos que se relacionan de manera inmediata con las enfermedades, especialmente con las enfermedades crónicas no transmisibles, de transformación de comportamientos generadores de pérdida de calidad de vida, o, por el contrario, de mejoramiento de la calidad de vida en contextos de enfermedad, y de modificación de comportamientos que se incluyen en el espectro amplio de las adicciones.

Se ha ofrecido en este escrito una definición que caracteriza de forma precisa el área de la IM; se sugiere que esta definición atienda a la referencia que necesariamente debe hacerse a dos aspectos centrales: 1) La referencia al proceso motivacional y a los factores que le subyacen en sus diversas fases, en calidad de variables intermediarias; este aspecto le brinda a la IM un espacio teórico muy amplio, que abarca campos como el de la percepción y las representaciones, la evaluación cognitiva, las atribuciones, la normatividad subjetiva, la toma de decisiones, y la autorregulación del comportamiento. Para el caso de aplicaciones de la IM en el campo de la salud, estas teorías adquieren cuerpo concreto en microteorías específicas acerca del comportamiento saludable, de gran auge en la actualidad (Conner, \& Norman, 2005). 2) La referencia a un sistema de formulación que permita identificar los mecanismos motivacionales que explican el estado actual de un comportamiento, sugerir hipótesis en busca de la modificación del comportamiento, y justificar el uso de procedimientos de evaluación y de intervención compatibles con la noción de IM. En el presente escrito se ha propuesto un sistema de formulación motivacional, derivado de otro sistema más amplio de formulación por niveles propuesto por Riso (2006) en el área de la terapia cognitiva. Particularmente el uso del sistema de formulación lleva a evitar la identificación de la IM con el uso de algún procedimiento particular, por importante que este haya sido a lo largo de su origen y evolución, como es el caso de la Entrevista Motivacional. De esta forma se pueden superar algunos inconvenientes atribuidos al uso del término «Intervención Motivacional» (Rollnick et al., 2002), al determinarse las condiciones precisas en que dicho uso puede hacerse, en función de la presencia o ausencia de una formulación que lo caracterice.

En el sistema de formulación propuesto se distinguen tres elementos esenciales: a) La descripción y el análisis de la situación problema, así como de los estímulos externos e internos sobresalientes que se relacionan funcionalmente con el comportamiento a modificarse en búsqueda de la solución del problema. b) El bloque central de percepción, evaluación, y valoración que realiza la persona en torno a las situaciones de estímulo relevantes, aspectos que se convierten en objetivo inmediato de modificación a través del uso de diversos procedimientos potenciales de intervención, y que permiten relacionar de forma inmediata a la IM con el proceso motivacional (Palmero et al., 2011). c) El resultado comportamental, descrito en términos de un proceso de afrontamiento emocional o instrumental. Estos tres elementos se pueden utilizar para que cumplan la función más importante de un sistema de formulación, cual es la de orientar la evaluación previa y posterior a la intervención, proponer un diagnóstico en términos de afectación de los factores psicológicos 
subyacentes a alguna de las fases propias del proceso motivacional correspondiente al comportamiento que se vaya a modificar, y establecer los objetivos directos de la intervención, así como seleccionar los procedimientos apropiados para cumplir esos objetivos.

La necesaria compatibilidad que cualquier intervención en el campo de la salud, independientemente de su naturaleza (médica, terapéutica, nutricional, física, odontológica, diagnostica, de enfermería, etc,), debe guardar con respecto a la naturaleza motivacional de toda acción humana, determina que los parámetros motivacionales deben ser tomados en cuenta con propiedad por todos los agentes que realizan intervenciones en este ámbito (los llamados «profesionales de la salud»). Eso no excluye que se pueda requerir de intervenciones especializadas para fomentar cambios específicos en el comportamiento saludable, las cuales demandan un conocimiento psicológico sobre el proceso motivacional y una habilidad particular, propios de una especialidad como la intervención motivacional en psicología de la salud. En esta revisión se ha ilustrado la situación actual de esta especialidad, particularmente en el desarrollo de protocolos susceptibles de aplicación en prácticas de educación para la salud recomendadas para la promoción de la salud, para la prevención universal de las enfermedades, y para la prevención selectiva e indicada de trastornos específicos.

Tomando los reportes de investigación incluidos en la base de datos Medline como una muestra bastante representativa de lo que acontece en el área de aplicaciones referente a la intervención motivacional en psicología de la salud se confirma que las mismas abarcan un período cercano a 25 años, el cual comenzó a inicios de la década 1990 con el uso de la IM para promover la moderación en el consumo de alcohol, haciéndose extensiva de manera progresiva al control en el consumo de otras sustancias como tabaco y marihuana, y luego a la solución de otras problemáticas propias del campo de la salud, dentro de las cuales sobresalen las referentes a la adhesión a los tratamientos, al control de las prácticas sexuales en situaciones de riesgo, y a la modificación de aspectos del estilo de vida que se relacionan con el origen y la evolución de enfermedades crónicas no transmisibles; llegando en la actualidad a consolidar un cuadro en el que la intervención motivacional en psicología de la salud aparece como una modalidad altamente recomendada en el contexto de la estrategia que la OMS denomina 4×4 (Lim et al., 2012; citados por OMS, 2013). Se trata de una estrategia que resalta la necesidad de controlar cuatro factores de riesgo (dietas malsanas, inactividad física, exposición al humo de tabaco, y uso nocivo de alcohol) para mejorar las altas cifras epidemiológicas asociadas a cuatro enfermedades no transmisibles (enfermedades cardiovasculares, cáncer, enfermedades respiratorias crónicas, y diabetes).

Actualmente se encuentran en pleno desarrollo las investigaciones que evalúan la efectividad de las diversas modalidades de IM para generar cambios en el estilo de vida, analizando la validez de los constructos teóricos o variables intermediarias sugeridas por las diversas microteorías de tipo motivacional acerca de la conducta saludable, y sometiendo a prueba su eficacia en planes de intervención breve que se proponen dos objetivos esenciales: 1) Fomentar la toma de decisiones de cambio de estilo de vida en personas que aún no tienen la intención explícita de realizar dichos cambios en un plazo temporal inmediato, para lo cual se resalta la intervención sobre algunos factores específicos de la dimensión psicológica inherente a la promoción y a la prevención (Flórez, 2007), tales como las expectativas de reforzamiento-resultado, las expectativas de autoeficacia, las normas subjetivas, y los balances decisionales. 2) Fomentar la elaboración de planes de autorregulación del comportamiento, cuando ya existe la intención explícita de modificación, con una disponibilidad inmediata a su implementación; estos planes se han venido denominando como «intenciones de implementación»(Gollwitzer, 1999). Aunque breves, esas intervenciones deben tener una duración que supera el límite de una sesión dedicada solamente al logro del propósito especificado en cada objetivo, superando el alcance de la recomendación de cambio o de una consejería breve típica (Rollnick et al., 2002) que hacen los profesionales de la salud, quienes usualmente se restringen al énfasis sobre la severidad de los daños potenciales y la vulnerabilidad ante los riesgos inherentes a algunos hábitos de comportamiento propios del estilo de vida de sus pacientes.

Las IM propiamente dichas, al margen de su brevedad, deben conducirse conforme a un esquema de formulación sustentado en alguna microteoría acerca del 
comportamiento saludable, con claridad acerca de los procesos de afrontamiento que se busca promover en la persona, de los mecanismos motivacionales que se busca afectar para fomentar la disponibilidad de la persona a hacerlo, y con especificación de los procedimientos de evaluación y de intervención que se siguen de manera programada y replicable para lograr esos objetivos. Esos procedimientos, de acuerdo con el estado actual de la investigación sobre intervención motivacional en psicología de la salud, deben cumplir con las condiciones sugeridas por los principios de la entrevista motivacional (Miller, \& Rollnick, 1991), pero deben además acoger los principios sobre autorregulación y autocontrol del comportamiento que se destacan al interior de modelos conceptuales que muestran gran auge y aceptación, como lo es en la actualidad el modelo teórico de la autodeterminación (Deci, \& Ryan, 2000; Ryan, \& Deci, 2000a, 2000b).

\section{Referencias}

Achtziger, A., \& Gollwitzer, P.M. (2008). Motivation and volition in the course of action. In: J.Heckhausen, \& H. Heckhausen (Eds.). Motivation and Action. Cambridge: Cambridge University Press, pp. 272-295.

Ajzen, I. (1985). From intentions to action: A theory of planned behavior. In: J. Kuhl, \& J. Beckman (Eds.). Action Control: From Cognitions to Behaviors. New York: Springer, pp. 11-39.

Belar, C., \& Deardoff, W. (1996). Clinical Health Psychology in Medical Settings. Washington: American Psychological Association.

Birk, L. (1973). Biofeedback: Behavioral medicine. New York: Grune, \& Stratton.

Craig, F., Hungerford, D., \& Dunn, C. (2005). Brief Motivational Interventions: An Introduction. Journal of Trauma-Injury Infection, \& Critical Care, 59(3), S21-S26.

Conner, M., \& Norman, P. (2005). Predicting health behaviour: A social cognition approach In: M. Conner, \& P. Norman (Eds.). Predicting Health Behaviour $2^{\text {nd }}$. Ed. London: Open University Press, pp. 1-27.

Davidson, D. (1992). Mente, mundo y acción. Barcelona: Paidós.

Deci, E. L., \& Ryan, R. M. (2000). The "what" and "why" of goal pursuits: Human needs and the self- determination of behavior. Psychological Inquiry, 11 , 227-268.

Dobson, K.S. (Ed.) (2010). Handbook of Cognitive-Behavioral Therapies. 3rd Edition. New York: The Guilford Press.

Dobson, K. S., \& Dozois, D. J. A. (2010). Historical and philosophical bases of the cognitive-behavioral therapies. In: K. S. Dobson (Ed.). Handbook of Cognitive-Behavioral Therapies. 3rd Edition. New York: The Guilford Press, pp. 3-38.

Fishbein, M., \& Ajzen, I. (1975). Belief, Attitude, Intention, and Behavior: An Introduction to Theory and Research. Reading, MA: Addison-Wesley.

Flórez, L. (2007). Psicología social de la salud: Prevención y promoción. Colombia: Manual Moderno.

Flórez-Alarcón (2007). Investigación y diseminación de CEMA-PEMA-P: Un programa con enfoque motivacional breve para la prevención primaria del abuso de alcohol en estudiantes. En: S.L. Echeverría, V.C. Carrascoza, \& M.L. Reidl (Compiladores). Prevención y Tratamiento de Conductas Adictivas. México: UNAM, pp. 237-272.

Flórez-Alarcón, L. (2013). Intervención motivacional: Una innovación cognitivo-conductual en psicología de la salud. Conferencia por Invitación. V Congreso Internacional de Psicología, Innovando las Intervenciones Psicológicas. Lima, Universidad Autónoma del Perú, 24 de Octubre de 2013.

Galvis, S.C. (2013). Diseño, implementación y evaluación de una intervención con medios audiovisuales, fundamentada en conceptos de motivación a la protección, para el fomento de la moderación en el consumo de alcohol en adolescentes. Bogotá: Tesis de Grado no Publicada, Programa de Maestría en Psicología, Universidad Nacional de Colombia.

Gil-Navarro, A. (2007). Aproximación al concepto de proceso en psicología. Psicología, Segunda Epoca, XXVI(2), 6-30.

Godoy, J.F. (1999). Psicología de la salud: Delimitación conceptual, En: M.A. Simón (Ed.). Manual de Psicología de la salud: Fundamentos, metodología y aplicaciones. Madrid: Biblioteca Nueva, pp. 39-65.

Gollwitzer, P.M. (1999). Implementation intentions: Strong effects of simple plans. American Psychologist, 54(7), 493-503. 
Grau-Abalo, J., \& Hernández-Meléndez, E. (2005). Psicología de la salud: Aspectos históricos y conceptuales. En: E. Hernández-Meléndez, \& J. Grau-Abalo (Eds.). Psicología de la salud: Fundamentos y aplicaciones. México: Universidad de Guadalajara, pp. 33-72.

Hazlett-Stevens, H., \& Craske, M.G. (2002). Brief cognitive behavioral therapy: definition and scientific foundations. En F. Bond, \& W. Dryden (Eds.). Handbook of brief cognitive behaviour therapy. West Sussex, England: Wiley, \& Sons, pp. 1-20.

Heckhausen, H., \& Gollwitzer, P.M. (1987). Thought contents and cognitive functioning in motivational versus volitional states of mind. Motivation and Emotion, 11, 101-120.

Heckhause, J., \& Heckhausen, H. (2008). Motivation and action: Introduction and overview. In: J. Heckhausen, \& H. Heckhausen (Eds.). Motivation and Action. Cambridge: Cambridge University Press, pp. 1-9.

Innis, N.K. (1999). Edward C. Tolman's purposive behaviorism. In: W. O'Donohue, \& R. Kitchener (Eds.). Handbook of Behaviorism. San Diego, CA: Academic Press, pp. 97-117.

Kahneman, D. (2003). A perspective on judgement and choice: Mapping bounded rationality. American Psychologist, 58(9), 697-720.

Kahneman, D. (2012). Pensar rápido, pensar despacio. Barcelona: Radom House Mondadori S.A.

Kazdin, A. E. (1983). Historia de la modificación de conducta. Bilbao: Editorial Desclée de Brouwer S.A.

Klinger, E., \& Cox, W.M. (2004). Motivation and the Theory of Current Concerns. In: W.M. Cox, \& E. Klinger (Eds.). Handbook of Motivational Counseling: Concepts, Approaches, and Assessment. England: John Wiley, \& Sons, pp. 3-27.

Lazarus, R.S., \& Folkman, S. (1984). Coping and Adaptation. In: W. Doyle Gentry (Ed.). Handbook of Behavioral Medicine. USA: The Guilford Press, pp. 282-318.

Lazarus, R.S., \& Folkman, S. (1986). Estrés y procesos cognitivos. Barcelona: Ed. Martínez Roca.

Lewin, K. (1978). La Teoría del Campo en la Ciencia Social. Buenos Aires: Paidós. Colección de escritos de Lewin editados por D. Cartwright.
Mahoney, M.J., \& Mahoney, K. (1981). Control Permanente de Peso: Una Solución Total al Problema de las Dietas. México: Trillas.

Martínez, E. (2012). El diálogo socrático en psicoterapia. Bogotá: SAPS.

Miller, W.R., \& Rollnick, S. (1991). Motivational interviewing: Preparing people to change addictive behavior. New York: Gilford Press.

Miller, W.R., Zweben, A., DiClemente, C.C., \& Rychtarik, R.G. (1995). Motivational Enhancement Therapy Manual: A Clinical Research Guide for Therapists Treating Individuals with Alcohol Abuse and Dependence. Rockville, MD: National Institute on Alcohol Abuse and Alcoholism, Project MATCH Monograph Series, Vol. 2.

O ’ Donohue, W., \& Kitchener, R.F. (1999). Introduction: The behaviorisms. In: W. O'Donohue, \& R. Kitchener (Eds.). Handbook of Behaviorism. San Diego, CA: Academic Press, pp. 1-13.

Ogden, J. (2003). Some problems with social cognition models: A pragmatic and conceptual analysis. Health Psychology, 22(4), 424-428.

OMS. (2004). Prevención de los trastornos mentales. Intervenciones efectivas y opciones de políticas. Ginebra: Organización Mundial de la Salud.

OMS. (2013). Enfermedades no transmisibles. Nota descriptiva. Recuperado de: http://www.who.int/ mediacentre/factsheets/fs355/es/

Organización Panamericana de la Salud (2007). Evaluation in health promotion. Principles and perspectives. Washington D.C.: OPS.

Palmero, F., Martínez-Sánchez, F., \& Fernández-Abascal, E. (2002). El proceso motivacional. En: F. Palmero, E. Fernández-Abascal, F. Martínez, \& M. Chóliz (Eds.). Psicología de la motivación y la emoción. Madrid: McGraw Hill/Interamericana de España, S.A.U., pp. 35-55.

Palmero-Cantero, F., Guerrero-Rodríguez, C., GómezIñiguez, C., Carpi-Ballester, A., \& Gorayeb, R. (2011). Manual de teorías emocionales y motivacionales. Castelló de la Plana (España): Publicacions de la Universitat Jaume I.

Persons, J.B., \& Davidson, J. (2010). Cognitive-Behavioral Case Formulation. In: K.S. Dobson (Ed.) Handbook 
of Cognitive-Behavioral Therapies. 3rd Edition. New York: The Guilford Press, pp. 172-195.

Potvin, L., Haddad, S., \& Frohlich, K. (2001). Beyond process and outcome evaluation: A comprehensive approach for evaluating health promotion programmes. In: I. Rootman, M. Goodstadt, B. Hyndman, D.V. McQueen, L. Potvin, J. Springett, \& E. Ziglio (Eds.). Evaluation in health promotion: Principles and perspectives. Copenhagen, Denmark: WHO Regional Publications, European Series No. 92, pp. 45-62.

Prochaska, J.O., DiClemente, C.C., \& Norcross, J.C. (1992). In search of how people change: Applications to addictive behaviors. American Psychologist, 47(9), 1102-1114.

Prochaska, J.O., Norcross, J.C., \& DiClemente, C.C. (1994). Changing for Good. New York: Avon Books.

Rachlin, H.M. (2000). The Science of Self-Control. Cambridge: Harvard University Press.

Reeve, J. (2003). Motivación y Emoción, 3ra. Ed.. México: McGraw-Hill Interamericana.

Restrepo, H.E (2001a). Agenda para la acción en promoción de la salud. En: H.E. Restrepo, \& H. Málaga (Eds.) Promoción de la Salud: Cómo Construir Vida Saludable. Bogotá: Editorial Médica Panamericana, pp. 34-55.

Restrepo, H.E. (2001b). Conceptos y definiciones. En: H.E. Restrepo, \& H. Málaga (Eds.) Promoción de la Salud: Cómo Construir Vida Saludable. Bogotá: Editorial Médica Panamericana, pp. 24-32.

Reynoso, L., \& Seligson, I. (2005). Psicología Clínica de la Salud: Un Enfoque Conductual. México: Editorial el Manual Moderno.

Riso, W. (2006).Terapia cognitiva:Fundamentos teóricos y conceptualización del caso clínico. Colombia: Editorial Norma S.A.

Rodríguez-Marín, J., \& Neipp-López, M. del C. (2008). Psicología social de la salud. En: J. RodríguezMarín, \& M. del C. Neipp-López (Eds.). Manual de Psicología Social de la Salud. Madrid: Editorial Síntesis S.A., pp. 15-39.

Rogers, R.W. (1975). A protection motivation theory of fear appeals and attitude change. Journal of Psychology, 91, 93-114.
Rollnick, S., Allison, J., Ballasiotes, S., Barth, T., Butler, Ch.C., Rose, G.S., \& Rosengren, D.B. (2002). Variations on a theme: Motivational interviewing. In: Miller, W.R., \& Rollnick, S. (Eds). Motivational interviewing: Preparing people for change, 2nd Ed. New York: The Guilford Press, pp. 270-283.

Rosenstock, L.M. (1974). Historical origins of the health belief model. Health Education Monographs, 2, 1-8.

Ryan, R. M., \& Deci, E. L. (2000a). Self-determination theory and the facilitation of intrinsic motivation, social development and well-being. American Psychologist, 55, 68-78.

Ryan, R. M., \& Deci, E. L. (2000b). Intrinsic and extrinsic motivations: Classic definitions and new directions. Contemporary Educational Psychology, 25, 54-67.

SAMHSA/CSAT (1999). Enhancing motivation for change in substance abuse treatment. Rockville (MD): Treatment Improvement Protocol (TIP) Series, No. 35. Substance Abuse and Mental Health Services Administratio (USA). Recuperado de: http://www.ncbi.nlm.nih.gov/books/NBK64967/

Schwartz, G.E., \& Weiss, S.M. (1978). Yale conference on behavioral medicine: A proposed definition and statement of goals. Journal of Behavioral Medicine, 1(1), 3-12.

Sutton, S. (2005). Stage theories of health behaviour. In: M. Conner, \& P. Norman (Eds.). Predicting Health Behaviour $2^{\text {nd }}$. Ed. London: Open University Press, pp. 223-275.

Tversky, A., \& Kahneman, D. (1974). Judgment under uncertainty: Heuristics and biases. Science, 185, 1124-1131.

Walliser, B. (2008). Cognitive Economics. Berlin: SpringerVerlag.

Weiner, B. (1985). An attributional theory of achievement motivation and emotion. Psychological Review, 92, 548-573.

Weinstein, N.D. (1988). The precaution adoption process. Health Psychology, 7(4): 355-386.

Weisz, J.R. Sandler, I.N., Durlak, J.A., \& Anton, B.S. (2005). Promoting and protecting youth mental health through evidenced-based prevention and treatment. American Psychologist, 6, 628-648. 


\section{Anexo 1}

\begin{tabular}{|c|c|}
\hline $\begin{array}{l}\text { Metodología } \\
\text { sistemática }\end{array}$ & $\begin{array}{l}\text { - Forma de proceder conducente a la consecución de objetivos ubicados a nivel de las variables cognitivas intermediarias propias del PM, que se localizan } \\
\text { entre el medio ambiente y el comportamiento del individuo, metodología que se sistematiza en un protocolo de formulación motivacional que orienta el } \\
\text { curso de la intervención. }\end{array}$ \\
\hline $\begin{array}{c}\text { Proceso } \\
\text { motivacional }\end{array}$ & $\begin{array}{l}\text { - Secuencia dinámica de fases conducentes a la ejecución de una acción con una intencionalidad que la persona decide de manera deliberada en función de } \\
\text { sus metas, representando un contexto más amplio que, pero incluyente de, la acción en sí misma, en interacción con el medio ambiente; contexto cuyoo } \\
\text { estado modifica la probabilidad de dicha acción, e incluye eventos que suceden antes, durante y después de la misma. }\end{array}$ \\
\hline
\end{tabular}

Factores subyacentes

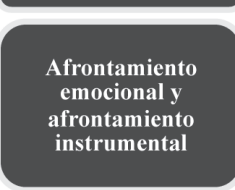

Acción

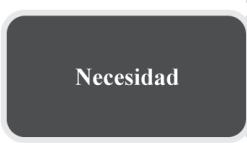

Persona

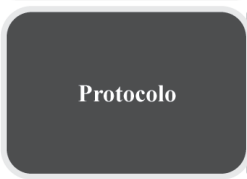

Intervención breve

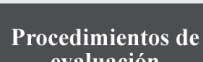
evaluación
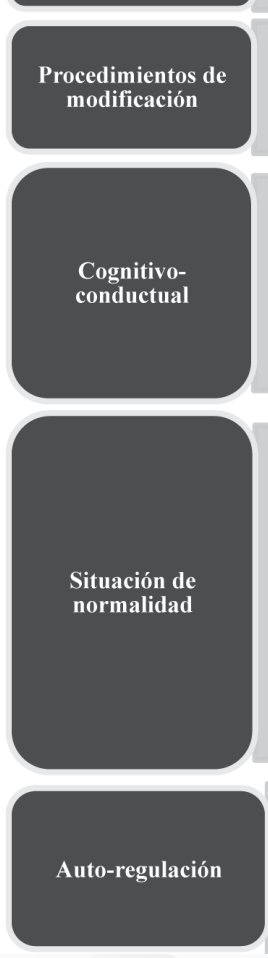

- Variables inferidas que se pueden localizar en las diversas fases del PM, a manera de variables intervinientes o de constructos hipotéticos en coherencia con la propuesta formulada en el modelo teórico de conductismo propositivo de Tolman (Innis, 1999), variables que permiten explicar los sucesos que acontecen en el ciclo motivacional y en el ciclo volitivo de una acción.

- Posición de inversión de esfuerzo que debe adoptar la persona para la realización de una acción en respuesta a situaciones de demanda marcadas por la presencia de una necesidad relevante, con el fin de obtener un resultado adaptativo favorable al logro de metas vitales. Este concepto es coherente con la propuesta cognitiva sobre afrontamiento que formulan Lazarus \& Folkman (1984). El afrontamiento es emocional cuando la acción de la persona tiene
como propósito fundamental modificar su propio estado emocional subjetivo, sin transformaciones en la situación del problema objetivo. Es instrumental como proposito fundamental modificar su propio estado emocional subjetivo, sin transformaciones en la situación del
cuando la acción tiene como resultado el logro de transformaciones objetivas favorables a la solución de un problema.

- Categoría central que conlleva a la adopción de una postura epistemológica que considera a la psicología como ciencia que estudia el comportamiento. La acción se refiere a la realización de un comportamiento o de un conjunto de comportamientos con un propósito deliberado por parte de la persona (Davidson, 1992).

- Estado inferido de la persona cuando se encuentra ante estímulos, externos e internos, que interpreta como una demanda para la realización de acciones conducentes al logro del equilibrio adaptativo. Se trata de un estado homólogo al de "tensión" propuesto en la Teoría del Campo (Lewin, 1978). La necesidad constituye el evento disparador que marca el inicio del PM, mediante la inducción de lo que usualmente se denomina "impulso" o "drive" en
psicología de la motivación (Palmero \& Cols., 2002). Se adopta la clasificación de necesidades fisiológicas, psicológicas y sociales (Reeve, 2003) para psicología de la motivación (Palmero \& Cols., 2002). Se adopta la clasificar
caracterizar los eventos que dan origen a la acción motivada de la persona.

- Organismo de la especie homo sapiens que en el transcurso de su evolución ontogenética ha aprendido un conjunto de comportamientos que le confieren su integridad individual estable y la diferencian de las demás, proceso de integración y diferenciación conducente a la formación de lo que en psicología se
denomina "personalidad".

- Conjunto objetivo y replicable de procedimientos de evaluación y de intervención utilizados en una aplicación particular de la IM. Constituyen la base que permite elevar la intervención a la categoría de procedimiento sustentado en la evidencia empírica, cuando pasa por fases de evaluación objetiva de su efectividad, eficacia, y eficiencia. La noción de protocolo exige que la intervención sea evaluada no solo en sus aspectos de impacto y de resultado, sino que sea abordada en una visión de programa que se desarrolla sucesivamente a la manera de una espiral que incluye y supera los productos que se obtienen a medida que se implementa, enriqueciéndose de forma progresiva, por lo cual debe someterse a las pruebas exigidas en la evaluación de programas
aplicables en el campo de la salud pública (Potvin. Haddad \& Frohlich, 2001: Organización Panamericana de la Salud, 2007). Una ilustración de protocolo aplicables en el campo de la salud pública (Potvin, Haddad \& Frohlich, 2001; Organización Panamericana de la Salud, 2007). Una ilustración de protocolo
de IM es el TIP 35 (sigla en inglés de Treatment Improvement Protocol), aplicado en la prevención selectiva e indicada del abuso alcohólico (SAMHSA de IM es el TIP 35 (sigla en inglés de Treatment Improvement Protocol), aplicado en la prevención selectiva e indicada del abuso alcohólico (SAMHSA/
CSAT, 1999).

- Carácter temporal de corta duración de la IM, cuyos protocolos se limitan a un número corto de sesiones con objetivos específicos discretos, que se circunscriben al manejo de variables propias de la fase motivacional del PM, en contraste con los objetivos de aprendizaje de habilidades que son propios de otras intervenciones terapéuticas que se concentran en la fase volitiva del PM.

- Instrumentos de medición del comportamiento en sus diversas manifestaciones cognitivas, fisiológicas y motoras, que permiten ponderar, mediante indicadores objetivos de diversos parámetros, el estado de las variables empíricas y de las variables intermediarias inferidas, para establecer de forma
válida y confiable su estado en diversos momentos, antes, durante y después de la intervención, lo cual permite evaluar el curso de su evolución, conforme a la metodología general establecida en los modelos de educación para la salud. - Conjunto amplio y variado de dinámicas específicas, cuya aplicación está regulada por una metodología coherente con los principios psicológicos que las
sustentan, implementadas de acuerdo con un protocolo objetivo para propiciar el cambio en los factores subyacentes al PM, incluido el cambio del comportamiento mismo. Algunos procedimientos cuyo uso es emblemático en la IMPS son la entrevista motivacional (Miller \& Rollnick, 1991), el diálogo socrático (Martínez, 2012), y los procedimientos de auto-control (Mahoney \& Mahoney, 1981; Rachlin, 2000). Se recomienda hacer una caracterización de estas dinámicas en términos de procesos de cambio, siguiendo la clasificación de procesos experienciales y conductuales propuestos en el marco del Modelo Transteórico de Cambio (Prochaska, Norcross \& Di Clemente, 1994).

- Corriente de teorización y de práctica psicológica que adopta lineamientos conceptuales, metodológicos y técnicos provenientes del enfoque cognitivo y del enfoque conductual en psicología. La inserción en esta corriente se justifica en la sustentación de la IM, dada la naturaleza multifactorial de las variables incluidas en el PM. El enfoque psicológico cognitivo enfatiza el análisis de las variables inferidas referentes al procesamiento de la información, a la manera de variables intervinientes y de constructos hipotéticos, como causas explicativas y no solo como manifestaciones del comportamiento (Dobson \& Dozois, 2010); este enfoque se expresa a través de diversas teorías y modelos de importancia actual en la ciencia psicológica y en la práctica psicoterapéutica, siendo de especial interés para la IMPS su impacto en la práctica de la intervención breve (Hazlett-Stevens \& Craske, 2002). El enfoque psicológico conductual enfatiza el análisis de las interacciones entre el comportamiento observable de los organismos y el medio ambiente, y también se expresa a través de diversas teorías y modelos (O’Donohue \& Kitchener, 1999) con gran impacto sobre la ciencia psicológica y la práctica de la modificación del comportamiento, siendo de especial interés para la IMPS sus aplicaciones en el campo del auto-control de la conducta (Kazdin, 1983; Rachlin, 2000).

- La IMPS supone ausencia de patología biológica o psicológica que impida la toma de decisiones autónomas de la persona, como pueden ser, a manera de ilustración, las patologías neuropsicológicas que afectan el funcionamiento cerebral requerido en los procesos normales de pensamiento, la patología inherente a una situación de dependencia de una sustancia, cuando el organismo requiere de la misma para su supervivencia por lo cual debe someterse a
intervenciones de rehabilitación en situaciones en las que prima el control externo sobre el interno, o en situaciones en las que la intervención se orienta intervenciones de rehabilitación en situaciones en las que prima el control externo sobre el interno, o en situaciones en las que la intervención se orienta
sobre la base del análisis de los acontecimientos propios de una psicopatologia. fundamentándose en inferencias acerca de las causas del comportamiento sobre la base del análisis de los acontecimientos propios de una psicopatologia, fundamentándose en inferencias acerca de las causas del comportamiento
anormal (ej. de un desorden de personalidad). La IM no constituye en sí misma una modalidad psicoterapéutica, pues se basa en el análisis del PM, tal anormal (ej. de un desorden de personalidad). La IM no constituye en sí misma una modalidad psicoterapéutica, pues se basa en el análisis del PM, tal
como este se aplica a la realización de cualquier acción, y no en el análisis del proceso cognitivo o conductual aplicado a una patología particular; sin como este se aplica a la realización de cualquier acción, y no en el análisis del proceso cognitivo o conductual aplicado a una patología particular; sin
embargo, puede ser utilizada como un complemento a la intervención psicoterapéutica para lograr objetivos como la adhesión al tratamiento o la práctica embargo, puede ser utilizada como un complemento a la intervencion psicoterapéutica para lograr objetivos como la adhesión al tratamiento o la práctica
de algún comportamiento específico que se encuentre en la esfera del control autónomo de la persona para contribuir a la mejoria de su calidad de vida. de algun comportamiento especíico que se encuentre en la esfera der control autonomo de la persona para contribuir a la mejoría de su calidad de vida.
En este sentido se trata de una intervención que tiene una característica preventiva predominante, susceptible de aplicación en contextos sociales de prevención universal, prevención selectiva, y prevención indicada de los trastornos físicos y mentales (OMS, 2004), o en contextos sociales de promoción de la salud (Restrepo, 2001a; 2001b), en contraste con los contextos clínicos propios del tratamiento y de la rehabilitación (Weisz, Sandler, Durlak \& Anton, 2005). Esto lleva a que la IMPS sea una alternativa más utilizada en la vertiente de la psicología de la salud conocida como psicologia social de la salud (Flórez, 2007a; Rodríguez-Marín \& Neipp-López, 2008) que en la conocida como psicología clínica de la salud (Belar \& Deardoff, 1996; Reynoso \& Seligson, 2005), en la cual es más practicada la intervención psicoterapéutica para el manejo de los desórdenes emocionales (ej. ansiedad, depresión) originados por la presencia de la enfermedad física.

- Primacia de la motivación autónoma sobre la motivación controlada, lo cual supone el acceso a niveles crecientes de internalización de la persona en el proceso regulatorio de la conducta. La internalización hace referencia a la apropiación consciente de la persona acerca de las expectativas (de reforzamiento, de resultado, de eficacia, etc.), los balances decisionales, las decisiones, los planes, las contingencias requeridas para la acción, y las atribuciones sobre las relaciones causales entre la acción y sus consecuencias, todo lo cual conduce al desarrollo creciente de su regulación autónoma para la acción. Esta regulación autónoma puede darse a un nivel diferencial que corresponda a la regulación introyectada, la regulación identificada, la regulación integrada, o la regulación interna de la conducta. Todos los términos que se han empleado en esta conceptualización de la auto-regulación se
utilizan en correspondencia con las definiciones y el uso que se hace de los mismos al interior de la Teoria de Auto-Determinación (Deci \& Ryan, 2000;
Ryan \& Deci, 2000a; 2000b). Ryan \& Deci, 2000a; 2000b). 


\section{Anexo 2}

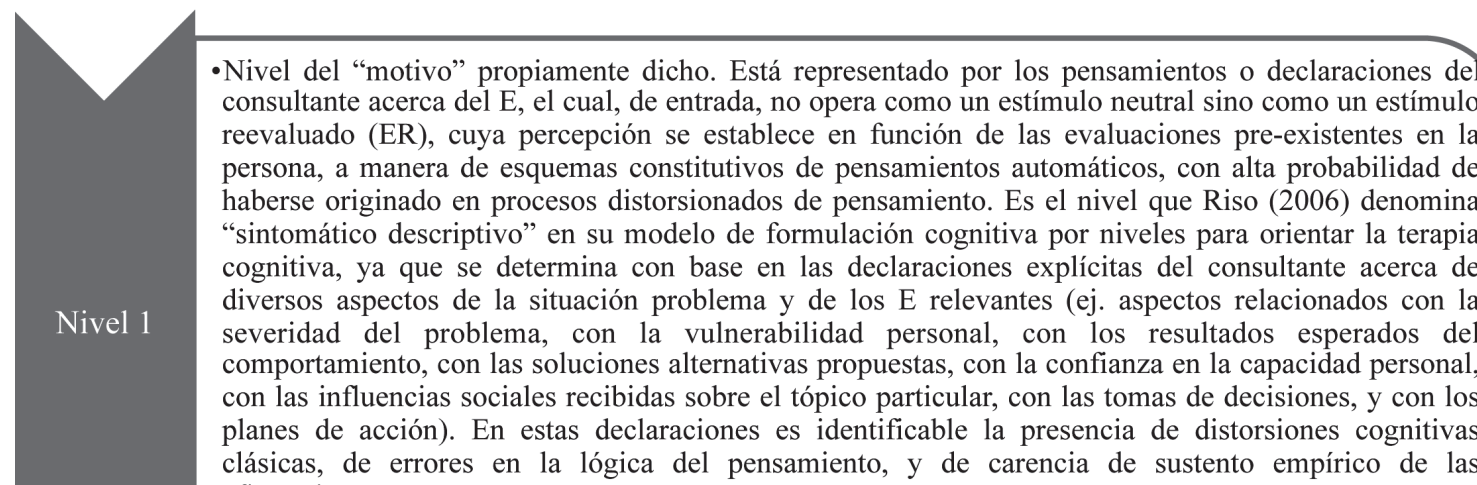
afirmaciones.

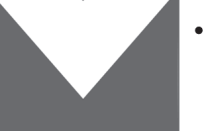

- Nivel de la "perspectiva", referente a los esquemas nucleares de vieja data y de gran arraigo en e sistema de pensamiento individual o cultural acerca de la situación problema. Se trata de la perspectiva que la persona, en su medio social y cultural, se ha forjado a lo largo de su vida en relación con aspectos centrales que marcan la percepción de la situación problema y de los E. Estos esquemas nucleares no tienen la accesibilidad propia de los pensamientos automáticos, por lo que deben ser identificados mediante un proceso de inferencia que realiza el interventor de manera inductiva (a partir de los pensamientos automáticos manifiestos), de manera deductiva (a partir de los modelos explicativos o microteorías existentes acerca de la situación problema), o ambas. Esos esquemas funcionan en forma de pensamientos rápidos constitutivos de sesgos o de heurísticos (Tversky \& Kahneman, 1974; Kahneman 2003, 2012) que dirigen el pensamiento y la toma de decisiones de la persona, con menor probabilidad de acierto en situaciones de incertidumbre, con sus consecuentes reacciones emocionales y conductuales, de conformidad con principios de economía cognitiva (Walliser, 2008). Riso (2006) considera que este nivel es de tipo preventivo, ya que debe agregarse a la modificación de los pensamientos automáticos del nivel 1, para restar probabilidad a la reincidencia de un comportamiento desadaptativo, o al resurgimiento de otros esquemas en forma de pensamientos automáticos alternativos a los ya modificados.

-Nivel del "sentido", representado por esquemas de tipo motivacional alusivos al sentido de vida de la persona, referente a su estructura motivacional. Se trata de representaciones acerca de lo que tiene mayor importancia en la vida de la persona, lo cual aporta elementos sustanciales para manejar el proceso motivacional de discrepancia, ya sea en una dirección que le permita a la persona establecer con claridad el grado en que el exceso o el déficit conductual afecta su logro de metas relevantes y de realización personal. Es por esta razón que Riso (2006) se refiere a éste como nivel promocional. 